\title{
NUMISMATICA Y EVIDENCIA ARQUEOLOGICA EN EL ALFAR ROMANO DE LA ALMADRAVA (SETLA - MIRAROSA - MIRAFLOR)
}

\author{
JUAN MANUEL ABASCAL \\ Universidad de Alicante \\ JOSEP A. GISBERT \\ Museo Arqueológico de Denia
}

\begin{abstract}
El enclave romano de La Almadrava (Setla-Mirarrosa-Miraflor) fue ocupado durante la época romana, especialmente entre los siglos I y III d.C. En él se fabricaron ánforas, ladrillos, cerámica común, etc. En este artículo se presenta el registro numismático hallado en sus excavaciones.

The roman site of La Almadrava (Setla-Mirarrosa-Miraflor) was occupated during the Roman period, especially between the I and III centuries a.D. Amphoras, bricks and common pottery were manufactured here. This paper presents the numismatic register found in its excavations.
\end{abstract}

El alfar romano de La Almadrava está situado en el término municipal de Setla-Mirarosa-Miraflor, junto al mar, al norte de la Punta de l'Almadrava (1). El área arqueológica se halla parcialmente cubierta por sedimentos de gravas aportados por el río Girona, que desemboca en sus inmediaciones; su altura sobre el nivel del mar oscila entre 0 y 5 metros y sus coordenadas se cruzan en el punto BD415057 del mapa 796 (30-31), escala 1: 50.000, del Servicio Geográfico del Ejército, quedando atravesado el paraje por la carretera de les Marines AP1324 (Fig. 1).

(1) Agradecemos al Prof. Pere Pau Ripollés (Univ. Valencia) su ayuda en la clasificación de algunos de los tipos monetales. Las abreviaturas empleadas se ajustan, siempre que ello es posible, a las de L'Annèe Philologique.
Un sector importante del yacimiento arqueológico se halla en la finca rústica conocida como Hort de Santonja, en donde ya en 1957, al transformar y adaptar los terrenos para el cultivo de regadío, aparecieron algunos materiales. Se dispone, así mismo, de noticias sobre el hallazgo en las inmediaciones de una necrópolis romana con ajuares de los siglos II y III d.C., y los abundantes restos cerámicos confirman la existencia de un establecimiento rural en actividad desde el siglo I d.C. hasta el siglo $\mathrm{V}$ d.C.

La creciente urbanización de la zona, y la necesidad de delimitar el yacimiento para la inserción de un área de protección en el planteamiento urbanístico, llevó a la realización de una prospección mecánica entre diciembre de 1984 y enero de 1985 . La detección de un área con estructura de hornos y de una densa escombrera de alfar aconsejó el inicio de 


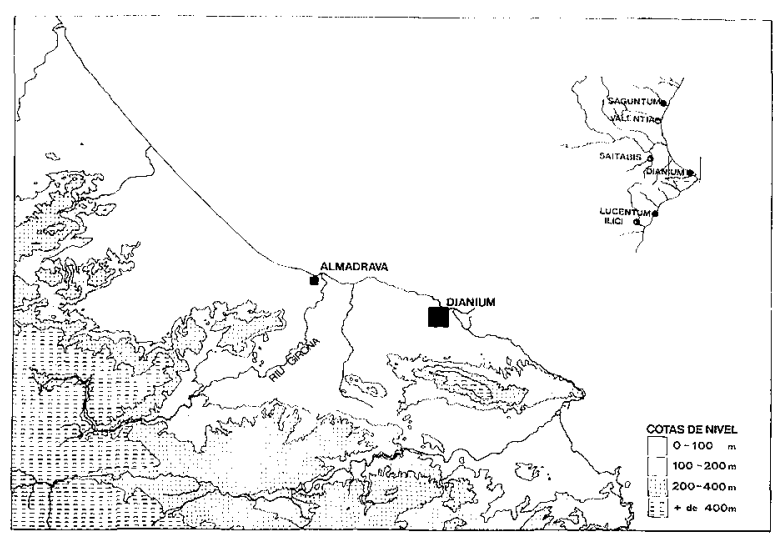

Fig. 1.- Plano que recoge parcialmente las comarcas de la Marina Alta y la Safor, territorium de Dianium, con situación del núcleo urbano y de la villa de La Almadrava (Setla-Mirarosa-Miraflor).

un proyecto de investigación arqueológica. Las campañas de excavación se han desarrollado entre 1986 y 1990, bajo la dirección de Josep A. Gisbert y autorizadas por la Dirección General del Patrimonio Artístico de la Consellería de Cultura, Educación y Ciencia de la Generalitat Valenciana. Los materiales se hallan depositados en el Museo Arqueológico de la ciudad de Denia (GISBERT 1988, passim).

\section{LA EVIDENCIA ARQUEOLOGICA}

\subsection{La distribución espacial}

El desarrollo de actuaciones arqueológicas en extensión, así como las diversas excavaciones de urgencia en sus proximidades, permiten esbozar parcialmente la organización y funcionamiento de este gran núcleo rural de época romana, que en su día perteneció al territorio de Dianium (Lám. 1).

Los trabajos de excavación sistemática se han desarrollado sobre una parcela de unos 9.000 metros cuadrados, y los resultados permiten observar que nos encontramos ante un complejo artesanal dedicado a la producción cerámica; en él se han individualizado hasta el presente cuatro talleres, situados en torno al área de hornos (Fig. 2).

El área excavada hasta la fecha es de unos 2.000 metros cuadrados, dos tercios de los cerca de 3.000 que debe alcanzar el conjunto artesanal a tenor de los indicios conocidos hasta el presente. La super-

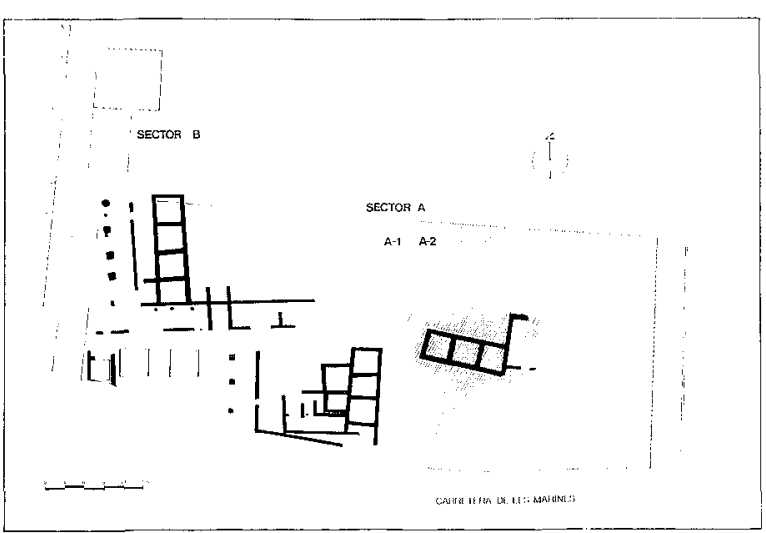

Fig. 2. - Plano del área excavada del yacimiento arqueológico de La Almadrava, con indicación de los sectores de excavación. A-1: sector artesanal o alfar: A-2: sector residencial.

ficie afectada por el trabajo cerámico sería superior, ya que a ello habría que añadir las zonas de extracción de arcilla y estructuras aisladas de hornos que se conocen fuera de los límites del área excavada. Parece razonable pensar que el complejo cerámico ocupe una superficie total de unos 6.000 metros cuadrados.

Los talleres, cuya superficie oscila entre los 200 y 300 metros cuadrados, presentan áreas cubiertas y otras no techadas; las primeras albergan el proceso de preparación de la arcilla y la fabricación de piezas, e incluyen las zonas de residencia; se comunican con las áreas descubiertas a través de estancias porticadas.

Al este del complejo alfarero se ha encontrado un edificio termal, provisto de hypocaustum de canales, así como diversas estancias contiguas que evidencian la existencia de un complejo de carácter residencial, sincrónico con diversas fases de actividad del alfar, cuya superficie supera los 1.800 metros cuadrados. Este sector se halla en proceso de excavación.

Un sondeo arqueológico de urgencia en una parcela contigua, a unos 150 metros al oeste del alfar, ha documentado más de quince sepulturas de inhumación en fosa, cubiertas mayoritariamente con tegulae, cuyos ajuares sitúan la deposición entre los siglos II y III d.C. Se trata, sin duda, de la necrópolis del establecimiento rústico.

El conjunto de actuaciones arqueológicas, así como la prospección arqueológica del área que nos ocupa, permiten precisar que el yacimiento 


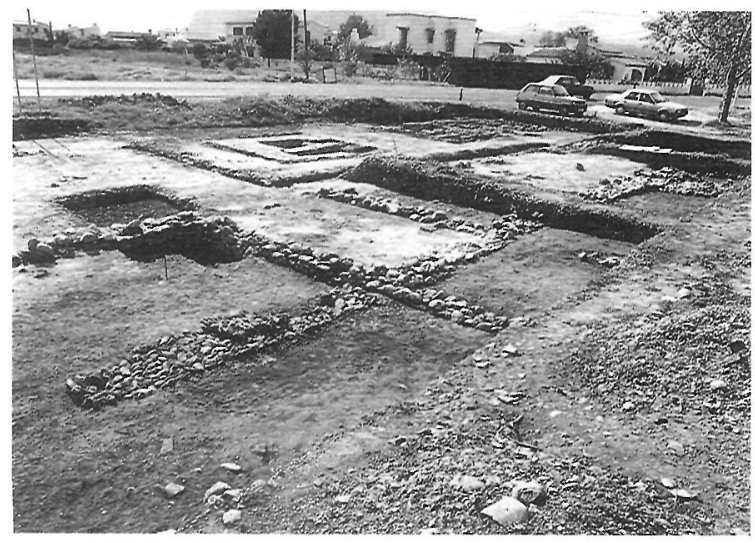

Lám. 1.- Almadrava (Setla-Mirarosa-Miraflor), campaña de 1986. En primer plano el taller I; al fondo, área de hornos.

-incluyendo área de trabajo, zona residencial y necrópolis - ocupa una superficie total de unos 22.000 metros cuadrados, 2'2 Has. Se trata, en consecuencia, de una de las villae o establecimientos rústicos más extensos del territorio de Dianium, tan sólo comparable con algunos núcleos litorales como el de la Partida Rafalcait (Gandía), a unos 15'5 km al noroeste, o el de la Punta del Arenal de Xàbia (MARTIN 1970, passim), a unos $20 \mathrm{~km}$ al sur de La Almadrava y a $8 \mathrm{~km}$ al sureste de Dianium (Lám. 2).

\subsection{Marco cronológico y propuesta de periodización. Arquitectura y registro arqueológico.}

La situación geográfica de este enclave, al norte de la desembocadura del río Girona, así como su peculiar topografía, con una amplia bahía al abrigo de las corrientes del sur, debieron favorecer el uso del lugar como fondeadero y área portuaria ya desde época tardorrepublicana. Así lo sugiere, al menos, el hallazgo en el registro arqueológico terrestre de materiales residuales como cerámicas de barniz negro «Campaniense $\mathrm{B} » \mathrm{y}$ «B-oide» $\mathrm{y}$ de «anforas itálicas».

Junto a estas evidencias, las campañas de prospección arqueológica subacuática (FERNANDEZ 1988, passim) desarrolladas èntre 1985 y 1987 confirmaron la existencia de un área de fondeadero frente al yacimiento, con abundantes ánforas de cronología tardorrepublicana, y permitieron localizar diversos pecios con cargamento de ánforas Dressel

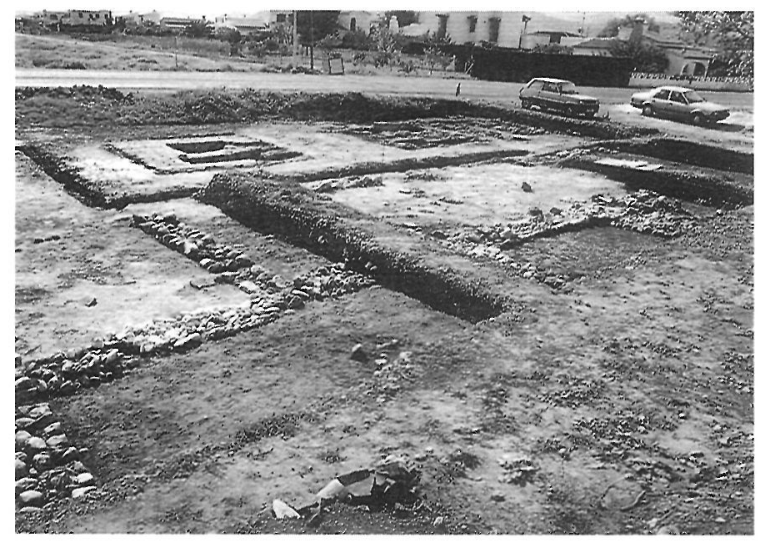

Lám. 2.- Almadrava (Setla-Mirarosa-Miraflor), campaña de 1986. Área entre los talleres I y II. Adosado al muro perimetral del taller I se observan vestigios de un horno de pequeñas dimensiones.

2/4, más tardías, ligadas al funcionamiento del alfar (FERNANDEZ y GISBERT 1990, passim).

Pese a disponer de abundantes materiales cerámicos de esta etapa tardorrepublicana, no se conocen en el área excavada estructuras arquitectónicas ligadas a estos hallazgos ni existen evidencias de las primeras décadas del siglo I d.C.

La primera fase de actividad del alfar se encuadra en el segundo tercio del siglo I d.C. A este momento corresponden los vestigios de canteras para la extracción de arcillas: grandes oquedades que perforan el suelo geológico, de naturaleza arcillosa, colmatadas con potentes estratos característicos que se interpretan como «escombreras de alfar». Las unidades estratigráficas que definen esta fase se caracterizan, en lo que se refiere a la producción de ánforas, por la presencia de los tipos Dressel 2/4 tarraconense y Dressel 20/26 (tipo Oliva 3) (GISBERT 1987, 104-118). Esta producción continúa hasta las últimas décadas del siglo I d.C.

La segunda fase se caracteriza por ser el momento en que el alfar se define en lo arquitectónico con elementos característicos. Los talleres de esta etapa son grandes edificios de planta rectangular, con una orientación norte-sur, dispuestos alrededor del área central de hornos que al parecer son comunes y que constituyen el eje arquitectónico del complejo.

El taller I mide 11'5 metros de longitud y 8' 5 de anchura, con una superficie de 98 metros cuadrados; posee cubierta a dos aguas (con tegulae) sustentada por pilares centrales de carga. Se trata de un tipo corriente en instalaciones de carácter industrial en todo el mundo romano, similar al de los ho- 


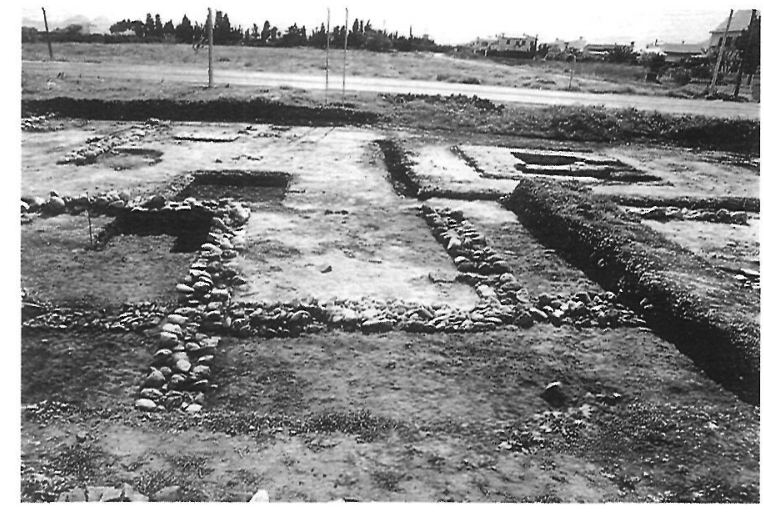

Lám. 3.- Almadrava (Setla-Mirarosa-Miraflor), campaña de 1986. Taller II; vista desde el sureste

rrea o almacenes. Este edificio, exento en un primer momento, se completa con un espacio porticado, también de planta rectangular, por el que se accede a un área descubierta o patio. La interpretación de las unidades estratigráficas de su interior permite reconstruir el proceso de elaboración de la producción del alfar y conocer la distribución del espacio en este tipo de edificios de uso industrial (Lám. 3).

El taller II presenta un edificio de características similares al originario del taller I, con compartimentación interna, aunque quizá menos reestructurado y modificado que aquél. El taller III presenta una distribución estructural y un proceso de ampliaciones prácticamente idéntico al del taller I. Se ha confirmado también la existencia de un taller IV, aunque el estado de los trabajos de excavación no permite hacer mayores precisiones.

La segunda fase, que coincide con la construcción de este complejo arquitectónico, se inicia en las primeras décadas del siglo II d.C. e incluye la producción durante todo este siglo. En cuanto a la producción de ánforas, que representa más del $80 \%$ de la actividad del centro cerámico, se caracteriza por la continuidad en la fabricación del ánfora vinaria de tipo Dressel 2/4 tarraconense, aunque con algunas variantes formales, y por la eclosión de un nuevo tipo de ánfora, hasta ahora ligada al área narbonense del sur de Francia y sólo esporádicamente presente en algún alfar del área catalana (GISBERT 1987, passim); se trata del ánfora de base plana de tipo Dressel 30, tipológicamente similar al ánfora «Gaulois 4», que se produce en el sur de Francia en la misma época en que se encuentra en el alfar que estudiamos (LAUBENHEIMER 1985, 391 y

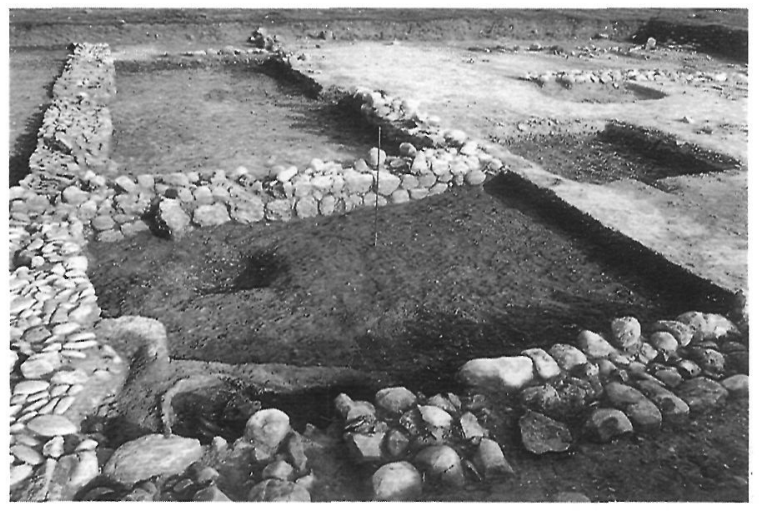

Lám. 4.- Almadrava (Setla-Mirarosa-Miraflor), campaña de 1986. Taller II; vista desde el norte.

1990, 106-110). En La Almadrava, a lo largo del siglo II d.C. esta producción experimenta algunos cambios que se traducen en modificaciones tipológicas de las ánforas, al tiempo que varían los datos porcentuales sobre su significación dentro del total de la producción.

La tercera fase se caracteriza por la remodelación total del conjunto arquitectónico vinculado a la producción alfarera. Al parecer, después de un hiato de difícil confirmación y del casi total derrumbe de las cubiertas y parte de los paramentos de los talleres de la fase anterior, se inicia la restauración total de los edificios, conservando la estructura externa de los muros perimetrales, aunque con una distribución espacial muy distinta; ahora se unifica la tipología de los edificios aislados de la fase anterior y se crean nuevas instalaciones anejas a los edificios originarios, destinadas al hábitat o vivienda de la mano de obra del centro artesano (Lám. 4).

En cuanto a la producción, esta última fase se caracteriza por el mantenimiento del tipo de ánfora Dressel 30, aunque con ciertas variantes formales que posibilitan el estudio de la evolución de este envase hasta el fin de su fabricación.

Esta tercera y última fase de la periodización del alfar es, sin duda, la que mejor define el proceso productivo, al tiempo que permite seguir las transformaciones que experimentó esta producción a lo largo de los tres primeros cuartos del siglo III d.C., pudiéndose situar el final de la actividad del centro alfarero en torno a los años 275-285 d.C.

En la periodización del yacimiento arqueológico se conocen también algunas fases de época bajoimperial, ligadas ahora a la continuidad y amplia- 
ción del sector residencial y a la reutilización de parte del sector alfarero como necrópolis.

\section{LOS HALLAZGOS MONETARIOS}

En el inventario que figura a continuación las piezas están descritas según la siguiente ficha modelo:

Número de orden. Emperador. Valor. Ceca. Cronología.

Anverso.

Reverso.

Exergo.

Peso / Diámetro / Posición de cuños. Número de registro en el inventario numismático del Museo Arqueológico de Denia (M.A.D.), fecha y año de hallazgo, unidad estratigráfica (Cfr. Tabla 6).

Bibibliografía.

En la consideración de los aspectos metrológicos debe tenerse en cuenta que el acusado desgaste de algunos ejemplares hace que su peso difiera del establecido para el tipo en los principales repertorios. Cuando la moneda está partida o le falta una porción significativa, su peso va encerrado en corchetes [].

\subsection{Siglos I y II}

\subsubsection{Acuñaciones hispanorromanas}

1.- As. LEPIDA - CELSA. 44/42 - 36/35 a.C. ?.

A.- [col vic iul lep]. Busto a derecha.

R. - [p salpa] M FVL[vi pr iivir]. Toro embistiendo hacia la derecha.

7'98/29/12. M.A.D. n 47, 7-89, 89-7-1.

Bib.: Vives CLX, 1-2; VILLARONGA 1979, n 896.

2.- AUGUSTO. As. CELSA. 36-27 a.C.

A.- II VIR - COL V I CELSA. Cabeza de ¿Augusto? a la derecha.

R.- L POMP B[ucco] y debajo L CORN FRO[nto]. Toro hacia derecha en el centro.

14'73/29/1. M.A.D. n० 29, 10-87, 88-3-1.

Bib.: Vives CLX, 9; Villaronga 1979, n 899.

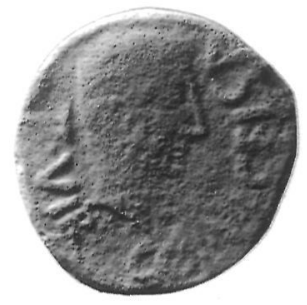

2

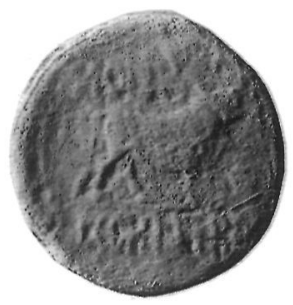

Lám. 5.- Catálogo n. ${ }^{\circ} 2$.

\subsubsection{Julio-Claudios}

3.- TIBERIO. As. ROMA. c. $22 / 23$ - 30 d.C.

A.- DIVVS AVGVSTVS PATER. Cabeza de Augusto radiada a izquierda.

R.- PROVIDENT S C. Altar con doble puerta y adornos superiores.

9'73/29/12. M.A.D. $\mathrm{n}^{\circ} 26,5-87,87-6-1$.

Bib.: RIC II 81.
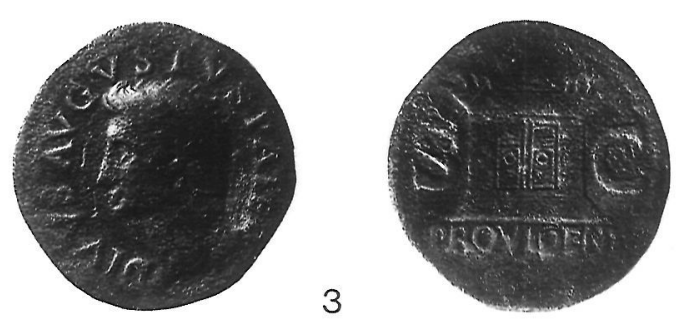

Lám. 6.- Catálogo n. ${ }^{\circ} 3$.

4.- CLAUDIO. Cuadrante. ROMA. 42.

A.- TI CLAVDIVS CA[e]SAR AVG. Modio.

R. - [po]N M TR P IMP P P [cos II] alrededor de S.C.

2’21/19/12. M.A.D. $\mathrm{n}^{\circ} 71,7-90,90-15-1$.

Bib.: RIC I 90

5.- CLAUDIO. As. ROMA ?. 41-50.

A.- TI CLAVDIVS CAESAR AVG P M TR P IMP. Cabeza desnuda a la izquierda.

R.- S C. Minerva con lanza y escudo hacia la derecha.

9'13/28/6. M.A.D. no 72, 7-90, 90-15-1.

Bib.: RIC I 100

6.- CLAUDIO. As. ROMA ?. 41-50.

A.- [---]ESAR AVGV[---]. Cabeza a la izquierda.

R.- [s] C. Minerva con lanza y escudo.

11'10/30/7. M.A.D. $n^{\circ}$ 64, 5-90, 90-7-1.

Bib.: ---

7.- CLAUDIO. As. ROMA ?. 41-50.

A.- [ti cla]VDIVS CAESAR AVG P M TR P IMP. Cabeza desnuda a la izquierda.

R.- CONSTANTIAE-AVGVSTI S C. Constantia en pie hacia la izquierda, sosteniendo lanza.

10’70/28/6. M.A.D. $\mathrm{n}^{\circ}$ 7, 6-86, 86-2-2.

Bib.: RIC I 95

\subsubsection{Flavios}

8.- DOMICIANO. As. ROMA. 86.

A.- IMP CAES DOMIT AVG GERM COS XII CENS PER

P P. Busto laureado a derecha.

R.- MONETA AVGVST S C. Moneta en pie hacia la izquierda sosteniendo balanza y cornucopia.

10'68/27/6. M.A.D. ${ }^{\circ} 22,10-86,86-3-118$.

Bib.: RIC II 327 (As) 
9.- DOMICIANO. As. ROMA. 87.

A.- [imp caes] DOMIT AVG GERM COS XIII [cens per p p]. Busto laureado a la derecha.

R. - [monet]A - AVG[ust s c.]. Moneta en pie hacia la izquierda sosteniendo balanza y cornucopia.

6'67/27/6. M.A.D. $n^{\circ} 32,11-87,88-3-1$.

Bib.: RIC II 354 b

10. - DOMICIANO. As. ROMA. 84-96.

A.- [---] GERM [---]. Cabeza laureada a derecha.

R.- Ilegible. Figura en pie hacia la izquierda.

$10^{\prime} 38 / 28 / 7$. M.A.D. $n^{\circ} 5,8-86,86-3-1$.

Bib.: -..

\subsubsection{Monedas no clasificables del siglo I}

11.- ATRIBUCION INDETERMINADA. As. ROMA. Primera mitad del siglo $\mathrm{I}$.

A.- [---] - AVGV[stus]. Cabeza laureada a derecha.

R. - Ilegible. Figura femenina con pátera y cornucopia.

$11^{\prime} 04 / 26 / 6$. M.A.D. $n^{\circ} 67,7-90,90-15-1$.

Bib.: ---

12.- ATRIBUCION INDETERMINADA. As. ROMA. Siglo I.

A. - Ilegible. Cabeza laureada a derecha.

R.- Ilegible.

4'93/26/---. M.A.D. n 3, 8-86, 86-3-1.

Bib.: ---
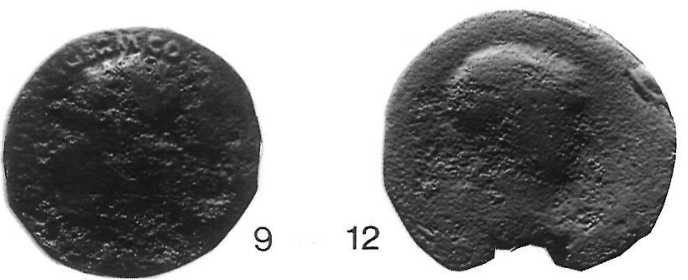

Lám. 7.- Catálogo n. ${ }^{\circ} 9$ y 12.

13.- ATRIBUCION INDETERMINADA. As. ROMA. Siglo I.

A.- Ilegible. Cabeza laureada a derecha.

R.- Ilegible.

7'97/26/--.. M.A.D. n 56, 8-88, 88-3-2.

Bib.: ---

14. - ATRIBUCION INDETERMINADA. As. ROMA. Primera mitad del siglo I.

A.- IMP [---]TVS

R.- [---]ATIA[---]. Perdido

[6'53]/27/---. M.A.D. n ${ }^{\circ} 57,8-88,88-3-2$

Bib.: ---

\subsubsection{De Trajano al final de los Antoninos}

15. - ADRIANO. Medallón. ROMA. 118.

A. - IMP [cae]SAR [traian] HADRIA - NVS [aug ?]. Cabeza laureada a derecha.

R.- P M TR P COS II; S C en exergo. Loba con gemelos a la izquierda.

59'48/42/12. M.A.D. $n^{\circ} 43,9-88,88-3-1$.

Bib.: COHEN n ${ }^{\circ} 1055$

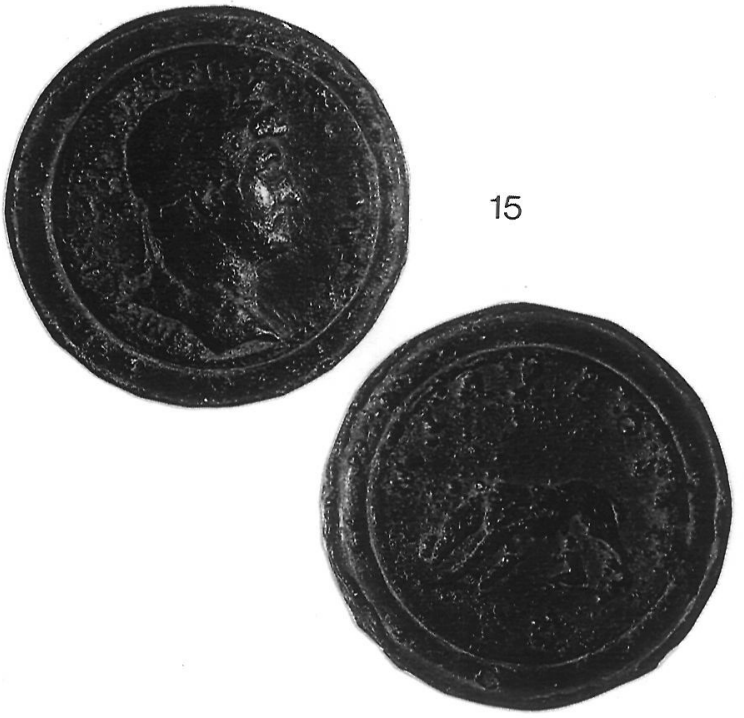

Lám. 8.- Catálogo n. ${ }^{\circ} 15$.

16.- ADRIANO. Sestercio. ROMA. 132-134.

A.- HADRIANVS AVGVSTVS. Busto a la derecha.

R. - FELICI[tati avg] COS III P P S C. Barco con proa a la izquierda.

24'21/32/6. M.A.D. $n^{\circ} 15,11-86,86-3-53$.

Bib.: RIC II 706

17.- ANTONINO PIO. As. ROMA. 139.

A.- ANTONINVS AVG PIVS PP. Cabeza laureada a la derecha.

R. - TR - POT - COS II; S C. Fortuna con cornucopia hacia la izquierda.

10'87/29/1. M.A.D. n ${ }^{\circ} 54,10-89,89-7-294$.

Bib.: RIC III 700 a = BMC 1164

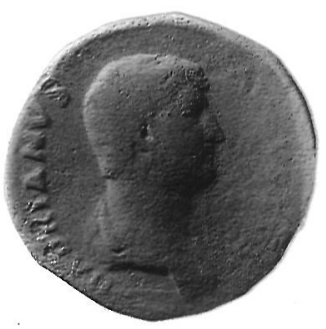

16
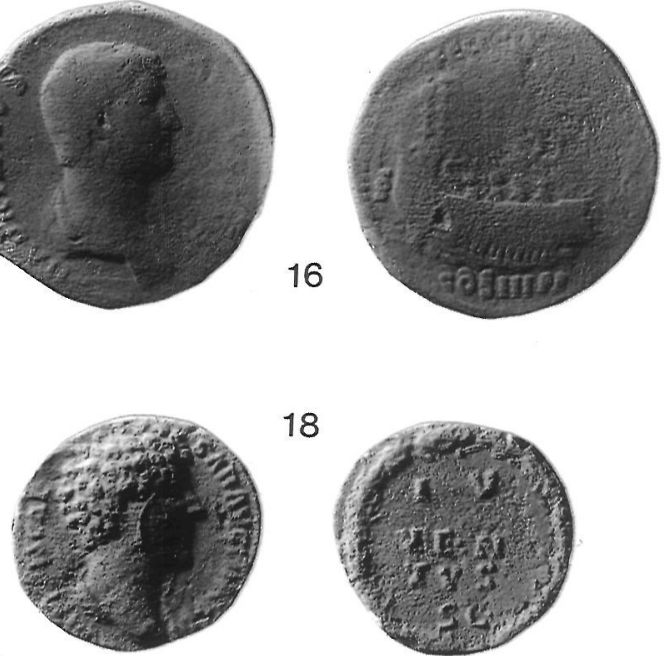

18

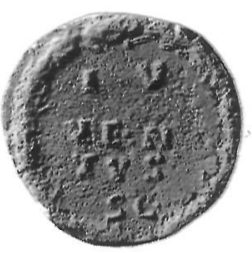

Lám. 9.- Catálogo n. ${ }^{\circ} 16$ y 18 
18.-ANTONINO PIO. Emisión a nombre de MARCO AURELIO. As. ROMA. 145.

A.-AVRE[1]IVS CAE - SAR AVG PII F COS. Busto barbado a derecha.

R. - IV / VEN / TVS / S C. en corona.

8'77/27/12. M.A.D. n' $19,11-86,86-3-65$.

Bib.: RIC III 1262

19.- MARCO AURELIO. Denario. ROMA. 168.

A.- M ANTONINVS AVG - ARM PARTH MAX. Cabeza barbada a derecha.

R. - FORT RED TR P XXII IMP V. Fortuna con cornucopia sentada hacia la izquierda.

Exergo: COS III.

2'40/18/6. M.A.D. no 62, 7-88, 88-3-2.

Bib.: RIC III 185

20.- MARCO AURELIO. Sestercio. ROMA. 161-180.

A.- [--- aureli]VS CAE - SAR AVG. Cabeza barbada a derecha. R.- [---]TAS S C. Emperador hacia la izquierda con victoriola sobre escudo.

22'51/32/12. M.A.D. n' $1,5-85,86-3-1$.

Bib.: ---

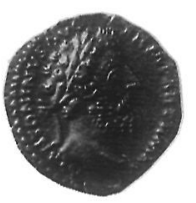

19
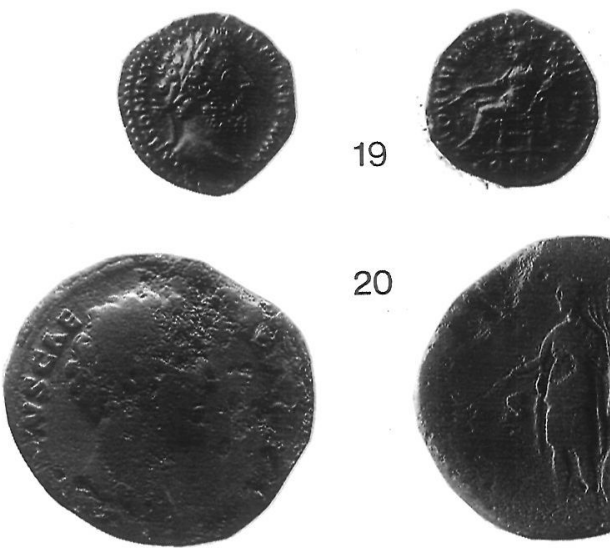

20

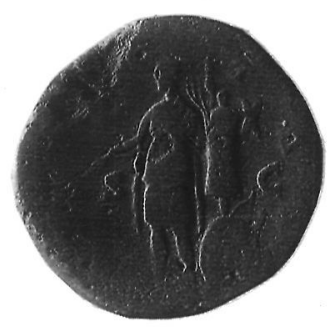

Lám. 10.- Catálogo n. ${ }^{\circ} 19$ y 20.

21.- LUCILLA. As. ROMA, $2^{\text {a }}$ emisión. 164-169.

A.- LVCILLA AVG[usta]. Busto a la derecha.

R. - HILAR - ITAS. Hilaritas con palma y cornucopia. A ambos lados: $\mathrm{S} \mathrm{C}$.

4'78/23/6. M.A.D. $n^{\circ} 20,11-86,86-3-66$.

Bib.: RIC III 1741

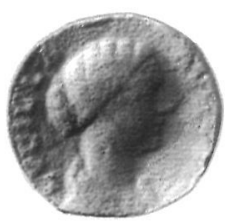

21

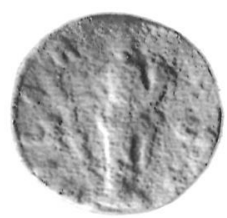

Lám. 11.- Catálogo n. ${ }^{\circ} 21$.
22. - FAUSTINA II. As. ROMA. 176-180.

A.- DIVA FAV[stina pia]. Cabeza femenina con pelo ondulado a la derecha.

R.- Creciente lunar con estrellas.

3'12/22/12. M.A.D. ${ }^{\circ} 103,7-86,85-2-11$ (sector B).

Bib.: RIC III 1714

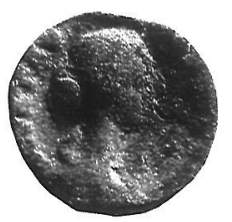

22

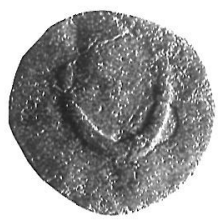

Lám. 12.- Catálogo n. ${ }^{\circ} 22$.

23.- COMODO. As. ROMA. 191-192.

A. - [1 ael] AVREL COM[m aug p fel]. Emperador con piel de león en la cabeza hacia derecha.

R.- HER - CVL / RO - MAN /AV - GV / S - C en cuatro líneas a ambos lados de una clava.

9'18/24/1. M.A.D. n ${ }^{\circ} 4,8-86,86-3-1$.

Bib.: RIC III 644

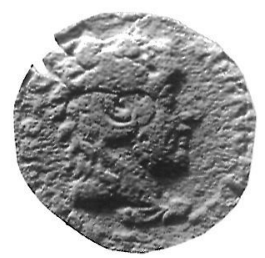

23

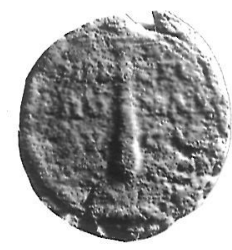

Lám. 13.- Catálogo n. ${ }^{\circ} 23$.

24. - ATRIBUCION INTDERMINADA. As ?. CECA LOCAL ORIENTAL. Segunda mitad del siglo II.

A.- Cabeza laureada y barbada a derecha.

R.- Diosa de la ciudad (Tyche ?) sentada sobre rocas. 8'75/26/9. M.A.D. n ${ }^{\circ} 14,11-86,86-3-53$.

Bib.: ---

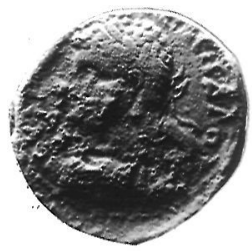

24

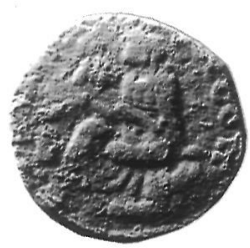

Lám. 14.- Catálogo n. ${ }^{\circ} 24$ 
25.- ATRIBUCION INTDERMINADA. Dupondio ?. ROMA.

Siglo II.

A.- IMP [---]. Cabeza laureada a derecha.

R.- [---] DES AVG. Loba con gemelos?.

Exergo: $\mathrm{S} \mathrm{C}$

15'45/---/---. M.A.D. n 68, 7-90, 90-15-1.

Bib.: ---

2.1.6. Monedas no clasificables de los siglos I y II

26. - ATRIBUCION INDETERMINADA. As. ROMA. Siglos I y II.

A.- Ilegible. Cabeza laureada a derecha.

R.- Ilegible. Figura en pie ?.

10'93/27/6. M.A.D. n 69, 9-90, 90-15-1.

Bib.: ---

27.- ATRIBUCION INDETERMINADA. As. ROMA. Siglos I y II.

A.- Ilegible. Cabeza laureada a derecha.

R. - Ilegible.

8'48/28/--.. M.A.D. n 65, 5-90, 90-7-1.

Bib.: ---

28.- ATRIBUCION INDETERMINADA. As. Ceca ?. Siglos I y II.

A.- Ilegible. Cabeza laureada a la derecha.

R. - Ilegible.

9'68/27/--. M.A.D. n 6, 9-86, 86-3-1.

Bib.: ---

\subsection{El siglo III}

\subsubsection{De la crisis del 193 a Galieno Augusto (260)}

29. - ALEJANDRO SEVERO. Sestercio. ROMA. 228.

A.- IMP SEV ALEXANDER AVG. Cabeza laureada a derecha.

R.- VIRTVS AVGVSTI S C. Virtus en pie hacia la derecha con lanza.

24'68/33/12. M.A.D. $n^{\circ} 8,12-86,86-3-44$.

Bib.: RIC IV.2 626 b

30.- ALEJANDRO SEVERO. As. ROMA. 231-235.

A.- [imp] ALEXANDER PIVS AVG. Busto laureado a derecha.

R.- [providentia aug s c]. Providentia con cornucopia hacia la izquierda.

8'20/28/12. M.A.D. n' 25, 6-87, 87-6-1.

Bib.: RIC IV.2 $644 \mathrm{~d}$

31.- GORDIANO III. Sestercio. ROMA. 238-239.

A. - [imp cae]S M A[nt gordian]VS AV[g]. Busto laureado, drapeado y con coraza a derecha.

R.- P M [tr p ii cos p p] S [c.]. Figura en pie.

[7'68]/32/1. M.A.D. $\mathrm{n}^{\circ} 40,12-87,88-3-224$.

Bib.: --.

32.- FILIPO I. Sestercio. ROMA. 247-249.

A.- IMP M IVL [ph]ILIPP[us aug]. Busto drapeado y lauredado a derecha.

R.- [ae]TERNITAS AVGG S C. Elefante en movimiento con conductor hacia la izquierda.

15'97/28/6. M.A.D. $\mathrm{n}^{\circ} 12,11-86,86-3-18$.

Bib.: RIC IV.3 167 a
33.- FILIPO II. Sestercio. ROMA. 244-247.

A.- M IVL PHILIPPVS CAES. Busto a la derecha.

R. - PRINCIPI I - VVENT. S C. Filipo en pie con globo y lanza. 21'44/30/1. M.A.D. n ${ }^{\circ} 41,12-87,88-3-236$.

Bib.: RIC IV.3 256 a
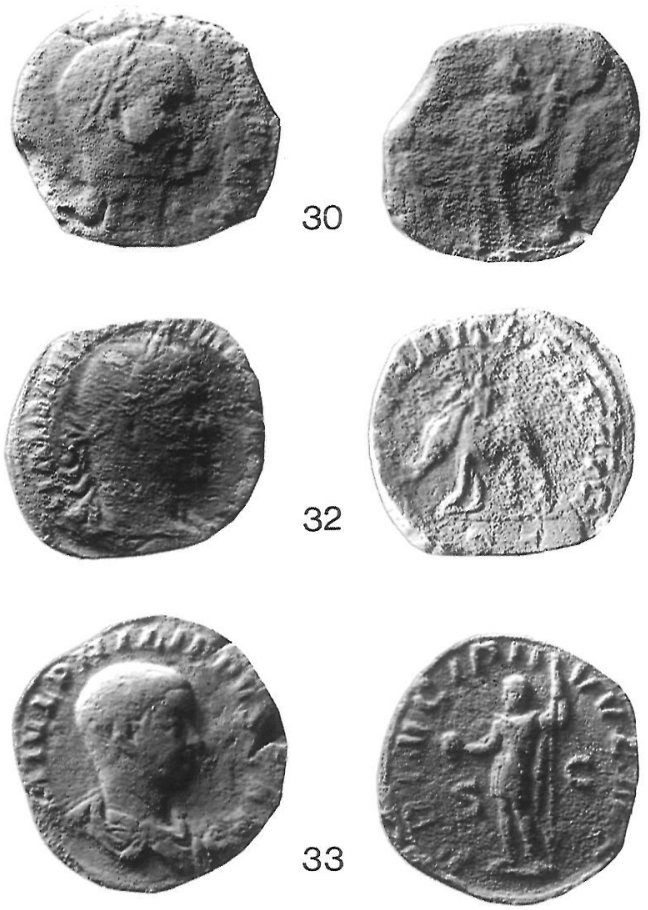

Lám. 15.- Catálogo n. ${ }^{\circ}$ 30, 32 y 33.

34. - TRAJANO DECIO. Radiado. ROMA. 249-250.

A.- IMP C M Q traianvs DECIVS AVG. Cabeza radiada a derecha.

R.- GENIVS EXERC ILLVRICIANI. Genius con pátera y cornucopia junto a estandarte.

3'96/21/12. M.A.D. n 44, 7-89, 89-7-2.

Bib.: RIC IV.3 18

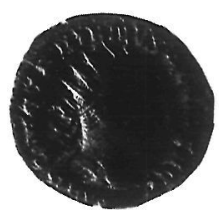

34
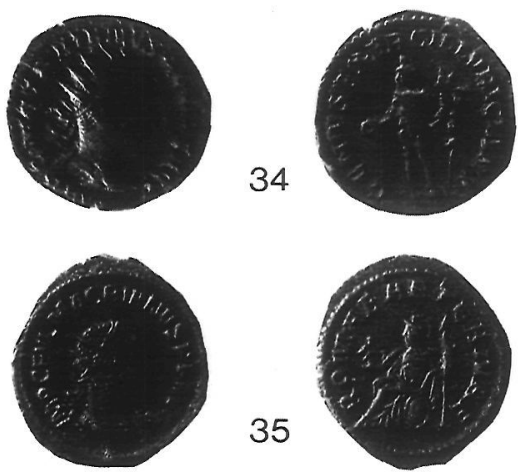

Lám. 16. - Catálogo n. ${ }^{\circ} 34$ y 35. 


\subsubsection{De Galieno a Diocleciano (260-284)}

35.- MACRIANO. Radiado. ANTIOQUIA. 260 d.C.

A. - IMP C FVL MACRIANVS P F AVG. Cabeza radiada a derecha.

R.- ROMAE AETERNAE. Roma, sentada sobre escudo, con lanza y victoriola.

3'99/22/6. M.A.D. n' $55,7-88,88-3-2$.

Bib.: RIC V.2 11

36.- GALIENO. Radiado. ROMA, emisión 'del séptimo consulado', of. $5^{\text {a }} \cdot 266$.

A.- GALLIENVS AVG. Cabeza radiada a derecha.

R. - VBERITAS AVG. Uberitas hacia la izquierda sosteniendo ramo de uvas y cornucopia.

Exergo:

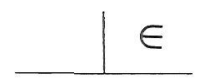

2'99/20/6. M.A.D. n 18, 11-86, 86-3-53.

Bib.: RIC ---; Canakkale 96

37.- GALIENO. Radiado. ROMA, emisión «del séptimo consulado», of. $2^{\text {a }}$. 266.

A.- GALLI[enu]S AVG. Cabeza radiada a derecha.

R.- ABVNDANT[ia aug]. Abundantia con cornucopia hacia la derecha.

Exergo:

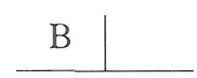

2'50/19/6. M.A.D. no 70, 8-90, 90-15-1.

Bib.: RIC V.1 157

38.- GALIENO. Radiado. ROMA, emisión «del séptimo consulado», of. $3^{\text {a }} \cdot 266$.

A.- GALLIENVS AVG. Cabeza radiada a derecha.

R.- AETER - NIT[as aug]. Sol hacia la izquierda sosteniendo globo.

Exergo:

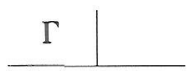

2'12/19/12. M.A.D. no 39, 8-87, 87-6-157.

Bib.: RIC V.1 160

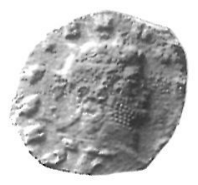

38
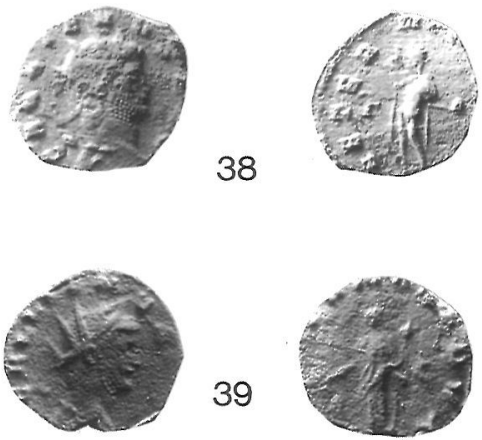

39

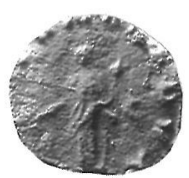

Lám. 17.- Catálogo n. ${ }^{\circ} 38$ y 39.
39. - GALIENO. Radiado. ROMA, emisión «del séptimo consulado», of. $5^{\text {a }}$. 266.

A.- GALLIENVS [aug]. Cabeza radiada a derecha.

R.- [for]TVNA REDVX. Fortuna con cornucopia hacia la izquierda.

Exergo:

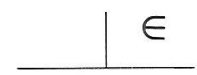

1'68/19/12. M.A.D. $\mathrm{n}^{\circ}$ 50, 7-89, 89-7-1.

Bib.: RIC V.1 193; Canakkale 120

40.- GALIENO. Radiado. ROMA, emisión 'del bestiario', 267-268.

A.- GALLIENV[s aug]. Cabeza radiada a derecha.

R.- SOLI CONS AVG. Pegaso al galope a derecha.

2'34/18/12. M.A.D. n 63, 4-90, 90-7-1.

Bib.: RIC ---; Canakkale 253

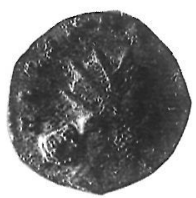

40

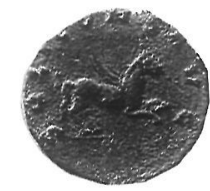

Lám. 18. - Catálogo n. ${ }^{\circ} 40$.

41.- GALIENO. Radiado. ROMA, emisión «del bestiario», of. $6^{\mathrm{a}}$. 267-268.

A. - [imp gallienus aug]. Busto radiado a derecha.

R.- [dianae cons aug]. Antílope hacia la izquierda.

Exergo:

2'63/18/11. M.A.D. no 51, 7-89, 89-7-1.

Bib.: RIC V.1 180

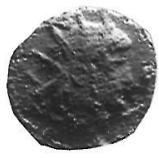

41

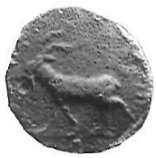

Lám. 19.- Catálogo n. ${ }^{\circ} 41$.

42.- CLAUDIO II. Radiado. ROMA, $1^{\text {a }}$ emisión. 268.

A. - [imp] C CLAVDIVS AVG. Busto radiado a derecha.

R.- LIBERALITAS [aug]. Liberalitas hacia la izquierda con tésera y cornucopia.

3'07/19/12. M.A.D. $n^{\circ} 17,11-86,86-3-53$.

Bib.: RIC V.1 57

43.- CLAUDIO II. Radiado. ROMA, $1^{\text {a }} / 2^{a}$ emisión, 268-269.

A. - IMP C CLAV[dius aug]. Busto radiado a derecha.

R.- MAR [ti pacif]. Marte hacia la derecha con lanza.

Exergo: ---

2'03/16/6. M.A.D. n० 35, 10-87, 88-3-2.

Bib.: RIC V.1 68

44.- CLAUDIO II. Radiado. ROMA, 2a emisión, of. 10ª 269.

A.- IMP C CL[audius aug]. Busto radiado a derecha.

R.- [fi]D[es] - EXER[ci]. Fides en pie sosteniendo dos insignias. 1'68/18/12. M.A.D. $n^{\circ} 27,7-87,87-6-1$.

Bib.: RIC V.1 34 


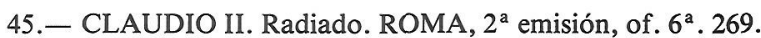

A. - [imp c cl]AVDIVS AVG. Busto radiado a derecha.

R.- [ae]QVITAS A[ug]. Aequitas con balanza y cornucopia hacia la izquierda.

Exergo:

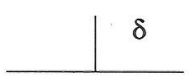

1'78/23/11. M.A.D. n' $11,11-86,86-3-18$.

Bib.: RIC V.1 14

46.- CLAUDIO II. Radiado. ROMA, 3ª emisión, of. 11² 269. A.- IMP CL[audi]VS AVG. Busto radiado a derecha.

R. - FIDE - S EXE - RCI. Fides en pie sosteniendo dos estandartes.

Exergo:

\section{XI}

1'53/20/5. M.A.D. no 31, 10-87, 88-3-1.

Bib.: RIC V.1 36

47.- CLAUDIO II. Radiado. ROMA, $3^{\text {a }} / 4^{a}$ emisión. 269-270.

A. - [imp cl]AVDIVS AVG. Busto radiado a derecha.

R.- PROVIDENT [aug]. Providentia con cornucopia junto a altar.

2'11/19/12. M.A.D. $\mathrm{n}^{\circ} 21,11-86,86-3-67$.

Bib.: RIC V.1 90

48.- CLAUDIO II. Radiado. ROMA, $4^{\mathrm{a}}$ emisión, of. $2^{\mathrm{a}}$. 269-270.

A.- - IMP CL[audius avg]. Busto radiado a derecha.

R.- VIRTV - S AV[g]. Soldado apoyado en escudo sosteniendo lanza.

Exergo:

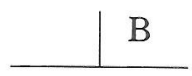

1'44/18/12. M.A.D. $n^{\circ} 10,11-86,86-3-18$.

Bib.: RIC V.1 111

49.- CLAUDIO II. Radiado. ROMA, $4^{\mathrm{a}}$ emisión, of. $5^{\mathrm{a}}$. 269-270.

A. - IMP CLAVDIVS A[ug]. Busto radiado a derecha.

R.- [for]TVNA REDVX. Fortuna con pátera y cornucopia.

Exergo:

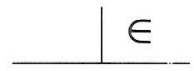

3'07/19/11. M.A.D. n ${ }^{\circ} 104,7-86,85-2-11$ (sector B).

Bib.: RIC ---

50.- CLAUDIO II. Radiado. SISCIA, of. $1^{\text {a }}$. 269-270.

A.- IMP CLAVDIVS AVG. Busto radiado a derecha.

R. - TEMP - [o]RVM FELI. Felicitas con caduceo y cornucopia. Exergo:

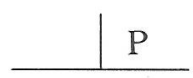

2'34/19/7. M.A.D. no 53, 6-89, 89-7-2.

Bib.: RIC V.1 192
51.- CLAUDIO II. Radiado. Ceca ?. c. 270.

A.- [divo] CLAV[dio]. Cabeza radiada a derecha.

R.- [consecratio]. Altar igual de ancho que alto.

1'78/18/5. M.A.D. ${ }^{\circ} 16,11-86,86-3-53$.

Bib.: RIC V.1 261.

52.- ClAUDiO II. Radiado. Ceca ?. c. 270.

A. - [divo claudio]. Cabeza radiada a derecha.

R.- CONSECR[atio]. Aguila.

0’96/14/6. M.A.D. no 9, 11-86, 86-3-18.

Bib.: RIC V.1 266.

53.- CLAUDIO II. Radiado. Ceca ?. c. 270.

A. - [divo cl]AVDIO. Cabeza radiada a derecha.

R.- CONSECRATIO. Aguila.

1'77/16/12. M.A.D. n' 28, 10-87, 88-3-1.

Bib.: RIC V.1 266.

54.- CLAUDIO II. Radiado. Ceca ?. c. 270.

A. - [divo cl]AVDIO. Cabeza radiada a derecha.

R.- [con]SECRAT[io]. Aguila.

2'11/17/10. M.A.D. n' 45, 7-89, 89-7-2.

Bib.: RIC V.1 266.

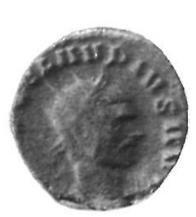

42
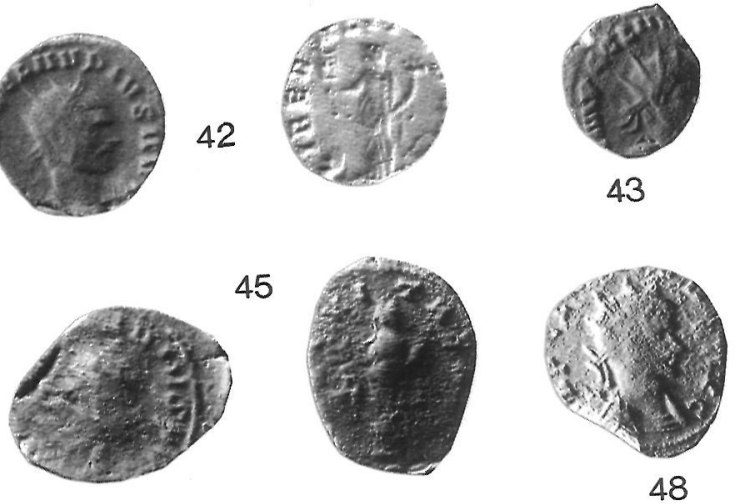

52
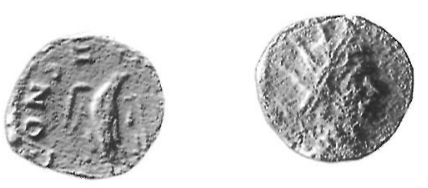

53

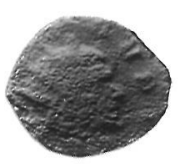

54

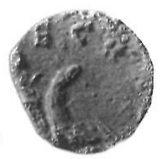

Lám. 20._ Catálogo ${ }^{\circ}{ }^{\circ} 42,43,45,48,52,53$ y 54 .

55.- VICTORINO. Radiado. TREVERIS. 269.

A.- IMP C VICTO[rinu]S P F AVG. Busto radiado a derecha. R.-SALV - S [aug]. Salus en pie sosteniendo pátera y cetro, con altar a la izquierda y serpiente. 2'26/19/6. M.A.D. nº 24, 7-87, 87-6-1.

Bib.: RIC V.1 67 . 


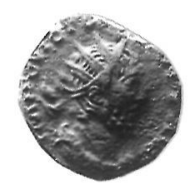

55

Lám. 21.- Catálogo n. ${ }^{\circ} 55$.

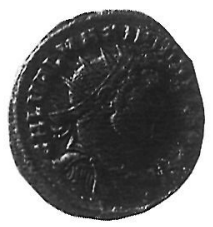

59

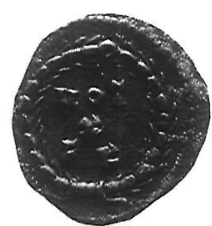

Lám. 24.- Catálogo n. ${ }^{\circ} 59$

60.- DIOCLECIANO. Fracción radiada. CARTAGO. 303. A.- IMP C DICOLETIAN[us p f ] AVG. Busto drapeado y radiado a derecha.

R.- VOT / XX en corona..

Exergo: $\quad F \mathrm{~K}$

3‘02/22/6. M.A.D. n. ${ }^{\circ}$ 38, 7-87, 87-6-149. Bib.: RIC VI 37a.
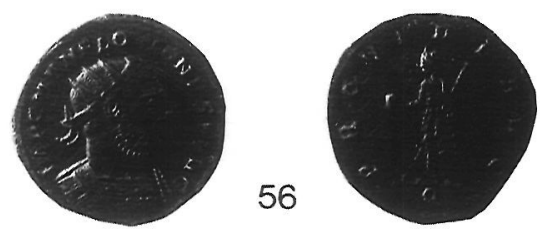

Lám. 22.- Catálogo n. ${ }^{\circ} 56$.

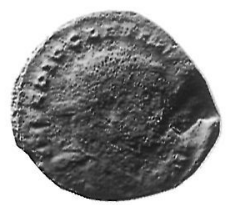

60

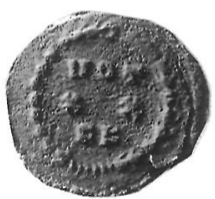

Lám. 25.- Catálogo n. ${ }^{\circ} 60$.

\subsection{El siglo IV}

- c.275.

A.- Ilegible. Cabeza radiada a derecha.

R.- [con]SECR[atio]. Aguila.

1'05/13/5. M.A.D. ${ }^{\circ} 46,7-89,89-7-2$.

Bib.: --.

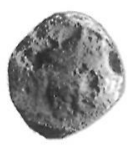

\section{7}

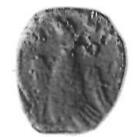

Lám. 23.—Catálogo n. ${ }^{\circ} 57$.

58.- ATRIBUCION INDETERMINADA. Radiado. Ceca ?. Segunda mitad del siglo III.

A.- [---] AVG. Cabeza radiada a derecha.

R.- [fides mili]TVM. Fides en pie sosteniendo dos estandartes.

1'84/15/6. M.A.D. n ${ }^{\circ} 102,7-86,85-2-1$.

Bib.: ---.

\subsubsection{I y II Tetrarquias (284-306).}

59.- MAXIMIANO. Fracción radiada. CARTAGO. 303.

A.- GAL VAL MAXIMIANVS NOB C. Busto drapeado y radiado a derecha.

R.- VOT / $\mathrm{X}$ en corona.

Exergo: $\quad \mathrm{F} \mathrm{K}$

3'13/22/6. M.A.D. $n^{0} 37,8-87,87-6-149$.

Bib.: RIC VI 35 b.

\subsubsection{De la proclamación de Constancio a la muerte de Constantino (324-337)}

61.- SERIES URBANAS. Nummus. ROMA, of. $4^{\mathrm{a}}$. 335-337.

A. - VRB[s - roma]. Cabeza con casco a la izquierda.

R.- Loba con gemelos bajo dos estrellas.

Exergo: R (hedera) Q

1'79/16/5. M.A.D. $\mathrm{n}^{\circ} 13,11-86,86-3-18$.

Bib.: LRBC I 575.

62.- CONSTANCIO II. Nummus. HERACLEA, of. $1^{\text {a }}$. 330-335.

A.- FL IVL CONSTANTIVS NOB C. Busto laureado y con coraza a derecha.

R.- GLOR - IA EXERC - [itus]. Dos estandartes.

Exergo: SMHA

2'37/18/12. M.A.D. $n^{\circ} 34,10-87,87-6-2$.

Bib.: LRBC I 911.

63.- POPULUS ROMANUS. Nummus. CONSTANTINOPLA, of. $11^{\text {a }}$. c. 337 ?.

A.- POP ROMANVS. Busto laureado con cornucopia hacia la izquierda.

R.- Puente sobre río.

Exergo: CONS

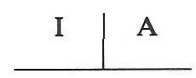

1'13/14/6. M.A.D. $n^{\circ}$ 2, 5-85, 86-3-1.

Bib.: RIC VIII 21 = LRBC I 1066. 
2.3.2. De la elevación de los hijos de Constantino como Augustos a la proclamación de Magnencio (337-350).

64. - CONSTANCIO II / CONSTANTE. Nummus. ROMA, of. 2. ${ }^{a} \cdot 337-341$.

A.- DN FL CONST [an---s aug]. Busto drapeado y diademado a derecha.

R. - [sec]VRI - TAS [r]EIP. Securitas con lanza y apoyada en columna.

Exergo: R (hedera) $\mathrm{S}$

1'44/15/12. M.A.D. n ${ }^{\circ} 52,6-89,89-7-2$.

Bib.: RIC VIII 9-13 = LRBC I 585-588.

65.- CONSTANTE. Nummus. ROMA, of. ?. 347-348.

A. - CONSTAN - [s p]F A[ug]. Busto drapeado y diademado a derecha.

R.- [vict]ORIAE DD A[uggq nn]. Dos Victorias enfrentadas sosteniendo cada una una pequeña corona..

Exergo: (hedera)

$$
[\mathrm{r}-\mathrm{-}]
$$

1'02/14/10. M.A.D. n० 30, 10-87, 88-3-1.

Bib.: RIC VIII $100=$ LRBC I 646
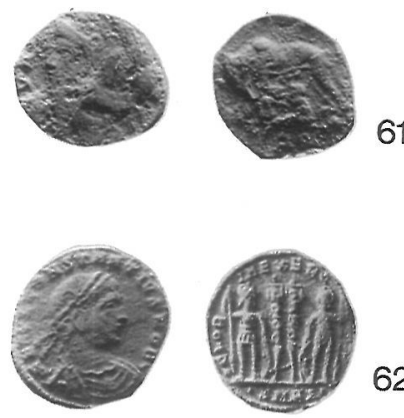

62
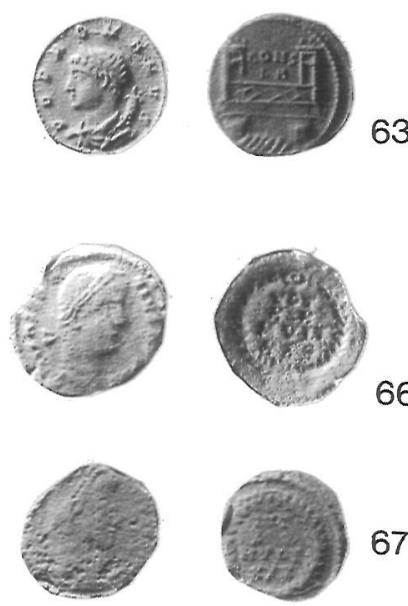

66

67
66.- CONSTANCIO II. Nummus. CYZICO, of. ?. 347-348 A.- DN CONSTAN - TIVS P F AVG. Cabeza laureada a derecha.

R.- VOT / XX / MVLT / XXX en corona.

Exergo: [smk-]

0'61/18/12. M.A.D. n ${ }^{\circ} 23,10-86,86-3-119$.

Bib.: RIC VIII $47=$ LRBC I 1305

67.- CONSTANCIO II / CONSTANTE. Nummus.

Ceca oriental. 347-348.

A.- Ilegible. Cabeza diademada a derecha.

R. - VOT / XX / MVLT / XXX en corona.

Exergo: ---

1’07/14/7. M.A.D. no 36, 10-87, 88-3-2.

Bib.: ---

\subsubsection{De la proclamación de Magnencio} a la muerte de Juliano (350-363)

68.- MAGNENCIO. AE 2 corto. Ceca ?. 351-353.

A. - [dn magnen - tius $p$ f aug]. Busto desnudo a derecha. Una letra $\mathrm{A}$ a la izquierda de ella.

R.- [vict dd nn aug et caes]. Dos Victorias sosteniendo un escudo en el que se lee VOT / V / MVLT / X en cuatro líneas. Exergo: [---]

1'84/16/6. M.A.D. n० 49, 7-89, 89-7-1.

Bib.: ---

69.- CONSTANCIO II. AE 3. CYZICO, of. $6^{\text {a }} \cdot 355-361$. A.- DN CONSTAN - TIVS P F AVG. Cabeza perlada a derecha.

R.- FEL TEMP - REPARATIO. Falling horseman 3b (2).

Exergo: SMKS

1'82/17/12. M.A.D. n 59, 8-88, 88-3-2.

Bib.: RIC VIII 110
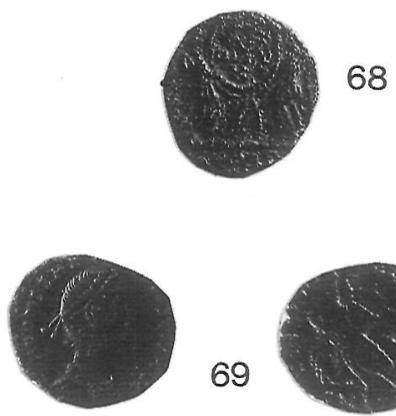

69

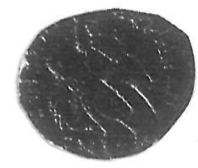

Lám. 27.- Catálogo n. ${ }^{\circ} 68$ y 69.

70.- CONSTANCIO II. AE 3. CYZICO, $6^{\mathrm{a}}$ of. 355-361. A. - DN [constan] - TIVS P F AVG. Cabeza perlada a derecha. R.- FEL TEMP - RE[paratio]. Falling horseman $3 b$. Exergo: SMKS 1'28/18/12. M.A.D. n' 66, 7-90, 90-15-1.

Bib.: RIC VIII 110

(2) Terminología de los tipos según Kent 1981, 38 s.

Lám. 26.- Catálogo n. ${ }^{\circ} 61,62,63,66$ y 67. 
71.- CONSTANCIO II / JULIANO. AE 4. Ceca ?. 355-361. A.- DN [---]. Cabeza perlada a derecha.

R. - SPES REI - [publice]. Emperador sosteniendo lanza y globo. Exergo: ---

1'58/14/12. M.A.D. $\mathrm{n}^{\circ} 61,3-88,88-3-268$.

Bib.: --.

72.-ATRIBUCION INDETERMINADA. AE 4. Ceca ?. 354-361.

A.- [---]ON[---]. Cabeza diademada a derecha.

R.- [fel temp reparatio], Falling horseman 3.

Exergo: ---

1'77/17/12. M.A.D. $n^{\circ} 60,9-88,88-3-436$.

Bib.: ---

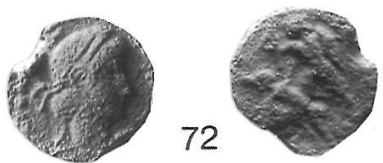

Lám. 28.- Catálogo n. ${ }^{\circ} 72$.

73.- ATRIBUCION INDETERMINADA. AE 3/4. Ceca ?. 354-361.

A. - Ilegible. Busto diademado a derecha.

R.- Ilegible. [Fel temp reparatio], Falling horseman 3.

Exergo: ---

1'15/17/---. M.A.D. no 33, 10-87, 88-3-2.

Bib.: ---

\subsubsection{De la proclamación de Valentiniano} a la cooptación de Teodosio (364-379).

74. - VALENTINIANO I / VALENTINIANO II. AE 3. Ceca ?. 364-392.

A.- DN VA[lentinianus --- aug]. Busto a la derecha.

R.- - [gloria ro-manorum], tipo LRBC II 6/8. Emperador hacia la derecha sosteniendo estandarte y arrastrando cautivo con la mano izquierda.

Exergo: --.

2'05/18/12. M.A.D. n 48, 7-89, 89-7-1.

Bib.: ---

\subsubsection{Moneda ilegible de la segunda mitad del siglo IV y principios del $V$}

75.- ATRIBUCION INDETERMINADA. AE 4. Ceca ?. Siglos IV-V.

A.- Ilegible.

R.- Ilegible.

0'50/13/---. M.A.D. n 101, 4-85, 85-2-1.

Bib.: --

\subsection{La circulación monetaria y los tipos}

El conjunto monetario de La Almadrava comienza en el tiempo con ejemplares de cecas hispanorromanas de la segunda mitad del siglo I a.C., cuyo reducido volumen apunta a su condición residual en el yacimiento.

Estas cecas hispanorromanas en La Almadrava están representadas únicamente por dos piezas de Celsa, una de ellas de época cesariana $\left(n^{\circ} 1\right)$ y la otra augustea $\left(\mathrm{n}^{\circ} 2\right)$. Probablemente debido a la situación septentrional del emplazamiento no aparecen aquí los característicos tipos de Carthago Nova e Ilici que caracterizan este tipo de emisiones en otros emplazamientos alicantinos; baste recordar que sobre el monetario del Museo Arqueológico Provincial de Alicante, Carthago Nova representa el $47^{\prime} 16 \%$ de las piezas de la segunda mitad del siglo I a.C. (LLOBREGAT 1968, 101); en Elche esta misma ceca constituye el $54^{\prime} 68 \%$ de los hallazgos hispanorromanos (LLOBREGAT 1968, 105 s.; GURT 1985, 60; RIPOLLES 1982, 430 s.), y en el Portus Ilicitanus ambas cecas alcanzan el 66'66 \% del total de las acuñaciones hispanorromanas (ABASCAL 1989, 19 y 26).

Excluidas las dos piezas citadas, anteriores al cambio de Era, el monetario de La Almadrava de los siglos I y II d.C. lo componen 26 piezas, el 34' 6 $\%$ del total de hallazgos; de ellas 12 corresponden al siglo I, 11 al siglo II y otras 3 , siendo con seguridad de los siglos I y II, no han podido ser clasificadas (Tabla 1).

En el monetario del siglo I d.C. anterior a los Flavios faltan las piezas de Augusto que, en mayor o menor medida, suelen aparecer en casi todos los enclaves de la costa mediterránea; se conserva una moneda de Tiberio y, como suele ser habitual, el reinado de Claudio es el mejor representado, con 4 ejemplares ( $\left.n^{\circ} 4-7\right)$ de los que $2\left(n^{\circ} 5\right.$ y 6$)$ son los característicos ases con reverso de Minerva con lanza y escudo que tan abundantemente fueron imitados por las cecas locales (CAMPO 1974, passim) (3), y que tanta difusión alcanzaron en las tierras valencianas (RIPOLLES 1980, 151). Por tipos, entre la muerte de César y el final de la dinastía julio-claudia se observa una neta superioridad de los ases ( 6 ejem-

(3) Puede verse además Bost y Pereira 1974, 167-181; Mateu y Llopis 1952, 49-53; Balil 1958, 25-29; Pereira et alii 1974, 219; Sutherland 1935, 24; Laffranchi 1949, 41; Giard 1970, 39-40. La opinión general hoy día es que no se trata de una moneda clandestina, sino que en cierto modo sustituye la falta de emisiones oficiales, y que la variedad de características en los diferentes enclaves hace pensar en una diversidad de talleres (BOST y PEREIRA 1974, 171; GIARD 1970, 39; GURT 1985, 67 s., n. ${ }^{\circ} 113$ con otra bibliografía). 
TABLA 1

\begin{tabular}{|c|c|c|c|c|c|}
\hline \multirow{2}{*}{\multicolumn{2}{|c|}{ SIGLOS I y II }} & \multicolumn{2}{|c|}{ N. ${ }^{\circ}$} & \multicolumn{2}{|c|}{ TOTAL } \\
\hline & & $\mathbf{A R}$ & $\mathrm{AE}$ & N. ${ }^{\circ}$ & $\%$ \\
\hline \multirow{2}{*}{$\begin{array}{l}\text { CECAS URBANAS } \\
\text { HISPANORROMANAS }\end{array}$} & AUGUSTO & & 1 & \multirow[b]{2}{*}{2} & \multirow[b]{2}{*}{7,1} \\
\hline & TIBERIO & & 1 & & \\
\hline \multicolumn{2}{|l|}{ JULIO-CLAUDIOS } & & & \multirow[b]{3}{*}{5} & \multirow[b]{3}{*}{17,8} \\
\hline TIBERIO & & & 1 & & \\
\hline CLAUDIO & & & 4 & & \\
\hline \multicolumn{2}{|l|}{ FLAVIOS } & & & \multirow[b]{2}{*}{3} & \multirow[b]{2}{*}{10,7} \\
\hline DOMICIANO & & & 3 & & \\
\hline \multicolumn{2}{|l|}{ SIGLO I indeterminado } & & 4 & 4 & 14,2 \\
\hline \multicolumn{2}{|l|}{ ADRIANO } & & 2 & 2 & 7,1 \\
\hline \multicolumn{2}{|l|}{ ANTONINOS } & & & \multirow[b]{6}{*}{7} & \multirow[b]{6}{*}{25} \\
\hline \multicolumn{2}{|l|}{ ANTONINO PIO } & & 2 & & \\
\hline \multicolumn{2}{|l|}{ MARCO AURELIO } & 1 & 1 & & \\
\hline \multicolumn{2}{|l|}{ LUCILLA } & & 1 & & \\
\hline \multicolumn{2}{|l|}{ FAUSTINA II } & & 1 & & \\
\hline \multicolumn{2}{|l|}{ COMODO } & & 1 & & \\
\hline \multicolumn{2}{|l|}{ SIGLO II indeterminado } & & 2 & 2 & 7,1 \\
\hline \multicolumn{2}{|l|}{ SIGLOS I y II indeterminado. } & & 3 & 3 & 10,7 \\
\hline \multicolumn{2}{|c|}{ TOTAL } & & & 28 & 100 \\
\hline
\end{tabular}

Tabla 1.- Hallazgos monetarios anteriores al siglo III en La Almadrava.

plares) sobre los cuadrantes (1 ejemplar); esto, unido a la escasa representación de las cecas locales, aparta el ritmo de circulación del yacimiento del conocido para otras zonas del País Valeciano.

El período flavio (69-96 d.C.) está representado en La Almadrava por 3 ejemplares de Domiciano, lo que arroja una cifra de 0'10 monedas/año; esta cantidad es notablemente inferior a la conocida para otros puntos de la región y de la Península, que en esos momentos registran cotas de 1'88 en Ampurias (RIPOLL et alii 1979, 50), 1'00 en Menorca (CAMPO 1979, 103), 1'92 en el conjunto del País
Valenciano (4) o 1'85 en Conimbriga (PEREIRA et alii 1974), dejando al margen los dos extremos representados por los 0,35 del Portus Ilicitanus (ABASCAL 1989, 31) y los 4'21 del Numario de la Universidad de Valencia (ARROYO 1984), explicable por el carácter de colección del conjunto. No se conservan en La Almadrava monedas del reinado de Trajano (98-117 d.C.), pero sí dos piezas

(4) Datos a partir de Ripollés 1980. Máximo aprovisionamiento hacia 74 d. C., según Arroyo 1980, 204. 
TABLA 2

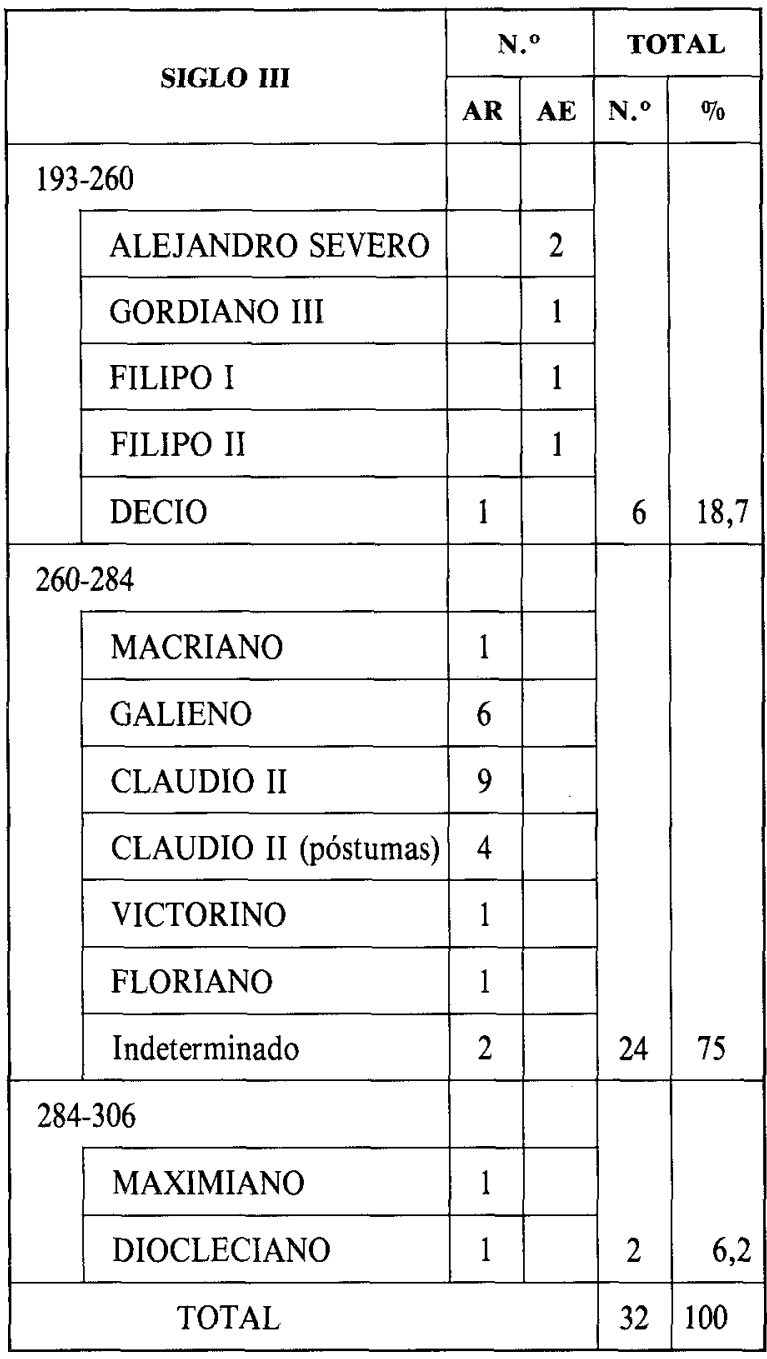

Tabla 2.- Hallazgos monetarios del siglo III en La Almadrava.

del de Adriano (117 - 138 d.C.), un sestercio y un medallón (vid. infra). Al período de los Antoninos (138 - 192) corresponden 7 piezas, que representan un 9'3 \% del total y arrojan una cifra de circulación de 0'12 monedas/año. Esta reducida cifra puede matizarse si, excluyendo las piezas de Antonino Pio y de Cómodo, consideramos que el período 161180 d.C. contiene 4 piezas, con una media de circulación superior al resto del siglo. La moneda más moderna del conjunto es el as de Cómodo $\left(\mathrm{n}^{\circ} 23\right)$ de los años 191-192 d.C.

La reducción de los aportes monetarios durante los dos últimos tercios del siglo II no es exclusiva de La Almadrava, sino que se registra en casi toda la Península. En el enclave, tras la moneda de Cómodo, se registra un vacío de hallazgos hasta la primera pieza del siglo III, un sestercio de Alejandro Severo $\left(\mathrm{n}^{\circ} 29\right)$, con el que se inaugura la circulación del siglo III.

El período 193-306 es, numismáticamente, el mejor representado en el alfar; incluye 32 ejemplares que suponen el 42'66 \% de los hallazgos totales, con una circulación media para todo el período de 0’28 monedas/año. Los extremos de este período los constituyen un sestercio de Alejandro Severo del año $228\left(n^{\circ} 29\right)$ y dos fracciones de Maximiano y Diocleciano acuñadas el año 303 d.C. en la ceca de Cartago ( $\mathrm{n}^{\circ} 59$ y 60 respectivamente).

Por encima de estos datos globales, como en la mayor parte de los enclaves, es posible realizar una nítida distinción entre la etapa anterior a la muerte de Valeriano (193-260) y la segunda mitad del siglo (260-306) (Tabla 2). Al primero corresponden 6 ejemplares (18'75 \% del siglo) y 26 al segundo (81'25 $\%$ ), porcentajes idénticos a los que recientemente hemos dado a conocer para el Portus Ilicitanus (ABASCAL 1989, 41). Como allí y en otros cientos de emplazamientos, el año 260 se puede considerar el primero de una etapa de aumento notable de la circulación que coincide con un permanente deterioro del numerario (CALLU 1969, 276).

La etapa anterior a la muerte de Valeriano significa en la política monetaria romana el fin del antiguo régimen apoyado en la plata y el bronce, sustituidos ahora por el radiado creado el año 215 (REECE 1986, 10; CALLU 1969, 478 y 481) (3), cuya pérdida de calidad se observa principalmente a partir del reinado de Galieno. En La Almadrava esta primera etapa está representada por seis piezas, cinco de bronce y un radiado de Trajano Decio que es el más moderno de los ejemplares del grupo ( $\left.{ }^{\circ} 34\right)$. El mantenimiento de las piezas de bronce hasta las cercanías de la década de los cincuenta es en La Almadrava evidencia de su homogeneidad con otros yacimientos valencianos, en los que el radiado comienza a imponerse, salvo excepciones (GONZALEZ y ABASCAL 1986, passim), a partir del año 253 (RIPOLLES 1980, 162).

(5) Denominación según Reece 1986, 10. 
Considerada en su conjunto, la etapa 193-260 d.C. arroja una media de 0,08 monedas/año, cantidad que oscila si dentro del grupo analizamos el antes y el después del año 238 d.C. Al primer grupo citado (193-238 d.C.) corresponden 2 piezas, con una media de monedas/año de 0,04; al segundo (238-260 d.C.) pertenecen 4 piezas, con una media de 0,18 monedas/año. Estas cifras son inferiores a las de otro contextos regionales: así, mientras La Almadrava proporciona una media de 0'08 monedas/año para la etapa 193-260, esta cifra se eleva a 0'17 en Santa Pola (ABASCAL 1989, 43) y en el conjunto del País Valenciano se obtienen medias de 0' 5 monedas/año para el intervalo 193-222 y de 2'32 para 222-253 (RIPOLLES 1980, 162); aún se elevan más estas cifras si abordamos únicamente el numario de la Universidad de Valencia, en donde alcanzan cifras de 2' 44 y 6'70 respectivamente.

Sin embargo, los reducidos valores de La Almadrava no son exclusivos del enclave ya que, al menos para el intervalo 193-222, conocemos aportes muy reducidos en Conimbriga, Clunia, Barcino, Portus Ilicitanus o Ampurias, por citar sólo algunos núcleos (ABASCAL 1989, 43).

En La Almadrava, y en el resto de los enclaves analizados en los últimos años, el volumen de numerario crece paulatinamente a partir del reinado de Alejandro Severo, todavía con notoria mayoría de especies en bronce, básicamente sestercios, para iniciar un aumento notorio desde el 260 , en que se incrementa notablemente el número de oficinas que emiten moneda.

La etapa 260-306 d.C. ofrece en La Almadrava 26 ejemplares, el 34' 6 de las monedas inventariadas, con una media de 0'56 monedas/año, muy superior a la de ningún otro momento en la vida del alfar; sus extremos son un infrecuente radiado de Macriano acuñado en Antioquía ( ${ }^{\circ} 35$ ) el 260 d.C. y las dos fracciones de Cartago ( ${ }^{\circ} 59$ y 60 ).

Dentro de esta etapa, que políticamente se sitúa entre los ascensos al poder de Galieno y Constantino, las desigualdades son evidentes. Mientras el intervalo $260-284$ ofrece 24 piezas ( $32 \%$ del total del alfar $=0$ '96 monedas/año), entre 284 y 306 sólo se registran 2 ejemplares (2'66\% del total $=0$ '08 monedas/año).

Estas cifras no son verdaderamente significativas consideradas globalmente, y las peculiaridades de la circulación monetaria en estos años sólo se observan en un análisis más pormenorizado (Tabla 3).

En efecto, los reinados de Galieno, Claudio II y Victorino (260-270 d.C.), sin considerar las piezas póstumas de Claudio II (vid. infra), concentran 16 monedas, entre las que no hay ninguna anterior al 266 d.C. Aún prescindiendo de este último dato, $\mathrm{y}$ ciñéndonos al decenio que abarcan los tres gober-

TABLA 3

\begin{tabular}{|c|c|c|c|c|c|}
\hline EMPERADOR & CECA & FECHA & EMISIÓN & $\mathbf{N} .^{\circ}$ & TOTAL \\
\hline GALIENO & ROMA & $\begin{array}{l}266 \\
267-8\end{array}$ & $\begin{array}{l}\text { «séptimo cos» } \\
\text { «bestiario» }\end{array}$ & $\begin{array}{l}4 \\
2\end{array}$ & 6 \\
\hline \multirow[t]{2}{*}{ CLAUDIO II } & ROMA & $\begin{array}{l}268 \\
268-9 \\
269 \\
269 \\
269-70 \\
269-70\end{array}$ & $\begin{array}{l}1 .^{a} \\
1 .^{a} / 2 .^{a} \\
2 .^{a} \\
3 .^{a} \\
3 .^{a} / 4 .^{a} \\
4 .^{a}\end{array}$ & $\begin{array}{l}1 \\
1 \\
2 \\
1 \\
1 \\
2\end{array}$ & \\
\hline & SISCIA & \multicolumn{2}{|l|}{$269-70$} & 1 & 9 \\
\hline $\begin{array}{c}\text { CLAUDIO II } \\
\text { (póstumas) }\end{array}$ & Local? & $\begin{array}{l}\text { c. } 270 \\
\text { c. } 270\end{array}$ & $\begin{array}{l}\text { Altar } \\
\text { Águila }\end{array}$ & $\begin{array}{l}1 \\
3\end{array}$ & 4 \\
\hline
\end{tabular}

Tabla 3.- Monedas de Galieno y Claudio II en La Almadrava. 
nantes, la media de circulación es la más alta de toda la vida del alfar (1'45 monedas/año), representando el 21'3 \% del total de hallazgos.

La explicación del proceso radica en el crecimiento progresivo del número de oficinas de la ceca de Roma a partir del Galieno, lugar del que proceden la mayor parte de los ejemplares que alcanzan las ciudades del occidente latino. De estas series, en $\mathrm{La}$ Almadrava están representadas la $4^{\mathrm{a}}$ emisión ('del séptimo consulado') y la $5^{\text {a }}$, conocida como emisión del "bestiario", término coloquial derivado de la presencia de motivos faunísticos en los reversos.

Este crecimiento en la acuñación de numerario redunda en que el número de monedas/año para el reinado de Galieno sea 0'66, significativo pero inferior al de otros emplazamientos (ARROYO 1982, 145; ABASCAL 1989, 49). Si consideramos globalmente los reinados de Galieno y Claudio II, el número de ejemplares es de 15 , con un aumento notable de la cantidad media de circulación.

El peso medio de las monedas de Galieno y Claudio en La Almadrava es la mejor evidencia de que nos encontramos ante moneda circulante. En efecto, frente a pesos que en la última emisión del «bestiario» oscilan entre los 2'58 y los 2'83 gramos para conjuntos resultado de tesaurización (CALLU 1969, 239), los ejemplares de Galieno de La Almadrava tienen un peso medio de 2'37 gramos, incluso inferior a los 2'43 del Portus Ilicitanus (ABASCAL 1989, 50). Del mismo modo, mientras en la ceca de Roma las emisiones de Claudio pierden paulatinamente peso, oscilando entre los 3'21 gramos de media para la primera y los 3'01 de la última, la media en La Almadrava es de 2'11 gramos debido al considerable deterioro de los ejemplares; sólo dos ejemplares, uno de la primera emisión $\left(n^{\circ} 42\right)$ y otro de la última ( $\left.n^{\circ} 49\right)$, con un peso de $3^{\prime} 07$ gramos, se mantienen en la banda de fluctuación de los tipos originales.

Especial interés revisten las monedas póstumas de Claudio II (CALLU 1969, 285; PEREIRA et alii 1974, 241; BOON 1978, 103; CENTENO 1981-82), los Divo Claudio, anotadas en el catálogo con una cronología circa 270 d.C. en razón de la fecha de defunción del Emperador. A partir de los trabajos de Callu sabemos hoy que este tipo monetal fue acuñado durante la década del 270 y que fue objeto de imitaciones locales, manteniéndose en circulación durante un buen número de años (CALLU 1974; CENTENO 1981-82, passim). Frente a cálculos que han llegado a sostener su mantenimiento en los cir- cuitos de numerario a comienzos del siglo IV, Callu sostiene que en el occidente latino el mayor volumen de este tipo de piezas debió circular entre los años 275 y 281 d.C. (CALLU 1969, 307, n. 5). En La Almadrava son cuatro los ejemplares pertenecientes a estas series, tres con reverso de águila y uno con el de altar ( $\left.{ }^{\circ} 51-54\right)$.

A la segunda mitad del siglo III pertenecen dos radiados de difícil filiación. El primero de ellos (n. ${ }^{\circ}$ 57) presenta un reverso de consecratio, no necesariamente perteneciente a un Divo Claudio; el segundo (n. $\left.{ }^{\circ} 58\right)$ ostenta un reverso de tipo fides militum, fácilmente identificable, pero que aparece en emisiones de casi toda la mitad del siglo, no siendo posible adscribirlo a un reinado específico.

La última moneda del intervalo $260-284$ es un radiado de Floriano acuñado en Ticinum el año 276 ( $\mathrm{n}^{\mathrm{o}} 56$ ), que da paso en La Almadrava a una fase sin restos de aprovisionamiento monetal, que termina el año 303 d.C., al que pertenecen dos fracciones radiadas de la ceca de Cartago ( $\mathrm{n}^{\circ} 59$ y 60 ).

Son éstas dos piezas emitidas para conmemorar los vicennalia de Diocleciano. La emisión comparte con las de Londres, Ticino y Roma el característico reverso de corona de laurel rodeando dos líneas de texto con la leyenda VOT / XX, pero añade el peculiar par de letras FK (Felix Karthago), omitiendo el número de la oficina emisora (SUTHERLAND 1967, 415). Aunque no son frecuentes los testimonios de esta emisión en el País Valenciano, en los últimos años han aparecido algunos ejemplares en las necrópolis de El Albir (Alfaz del Pi, Alicante) (ARROYO 1987-88, 153-172), y se conservan ejemplares inéditos, que pronto serán dados a conocer, procedentes de las excavaciones en el Portus Ilicitanus.

En líneas generales, el aporte de numerario de La Almadrava para esta segunda mitad del siglo III coincide con el observado en núcleos como Barcino, Tarraco, Ilici o el Portus Ilicitanus, cuyos patrones de circulación han permitido recientemente distinguir un «modelo urbano mediterráneo hispánico» (MEDRANO 1990, 70 s.).

En esta etapa final del siglo III se cierra la fase de actividad de los alfares de La Almadrava, por lo que el monetario debe datar sus últimos momentos de vida. Este límite cronológico debe fijarse entre los radiados de la segunda mitad del siglo III y las fracciones de la ceca de Cartago. Nominalmente, el más moderno de los primeros es uno a nombre de Floriano, datado en el año 276; junto a él 
hay que situar los cuatro ejemplares de Divo Claudio y su dilatada permanencia en los circuitos monetales. En cualquier caso, no se debe suponer para estas piezas un aporte posterior al $284 / 285$, con lo que el intervalo 284-303 representa un hiato en el que hay que suponer que el alfar ya ha sido cerrado. Por delante de este intervalo, las monedas de Galieno, Claudio II, Victorino y Floriano, indican el momento de plena actividad; por detrás, exceptuadas las dos piezas del mismo año 303, persiste la ausencia de numerario hasta comienzos de la década del 330 , en que aparecen las primeras piezas correspondientes a la nueva etapa residencial del lugar.

El siglo IV en La Almadrava (Tabla 4) no tiene ninguna trascendencia numismática: 15 piezas, el 20

TABLA 4

\begin{tabular}{|c|c|c|c|}
\hline \multirow{2}{*}{ SIGLO IV } & \multirow[b]{2}{*}{$\mathbf{N} .^{\circ}$} & \multicolumn{2}{|c|}{ TOTAL } \\
\hline & & N. ${ }^{\circ}$ & $\%$ \\
\hline \multicolumn{2}{|l|}{$324-337$} & \multirow[b]{4}{*}{3} & \multirow[b]{4}{*}{20} \\
\hline CONSTANCIO II & 1 & & \\
\hline SERIES URBANAS & 1 & & \\
\hline POPULUS ROMANUS & 1 & & \\
\hline $337-350$ & & \multirow[b]{4}{*}{4} & \multirow[b]{4}{*}{26,6} \\
\hline CONSTANCIO II & 1 & & \\
\hline CONSTANTE & 1 & & \\
\hline CONST. II - CONST. & 2 & & \\
\hline $350-363$ & & \multirow[b]{4}{*}{6} & \multirow[b]{4}{*}{40} \\
\hline MAGNENCIO & 1 & & \\
\hline CONSTANCIO II & 3 & & \\
\hline Indeterminado & 2 & & \\
\hline $364-378$ & & \multirow[b]{2}{*}{1} & \multirow[b]{2}{*}{6,6} \\
\hline VALENTINIANO I - II & 1 & & \\
\hline Indeterminado & 1 & 1 & 6,6 \\
\hline \multicolumn{2}{|l|}{ TOTAL } & 15 & 100 \\
\hline
\end{tabular}

Tabla 4.- Hallazgos monetarios del siglo IV en La Almadrava.
$\%$ de los hallazgos, alejan notablemente nuestros datos de los conocidos para otras zonas del País Valenciano, en donde normalmente los hallazgos del siglo IV alcanzan o superan el $50 \%$ de los valores totales. La razón está es la condición residual del conjunto, teniendo en cuenta que La Almadrava ya no es ahora un centro de actividad artesanal, y que el lugar conoce en este período tan sólo la ocupación residencial.

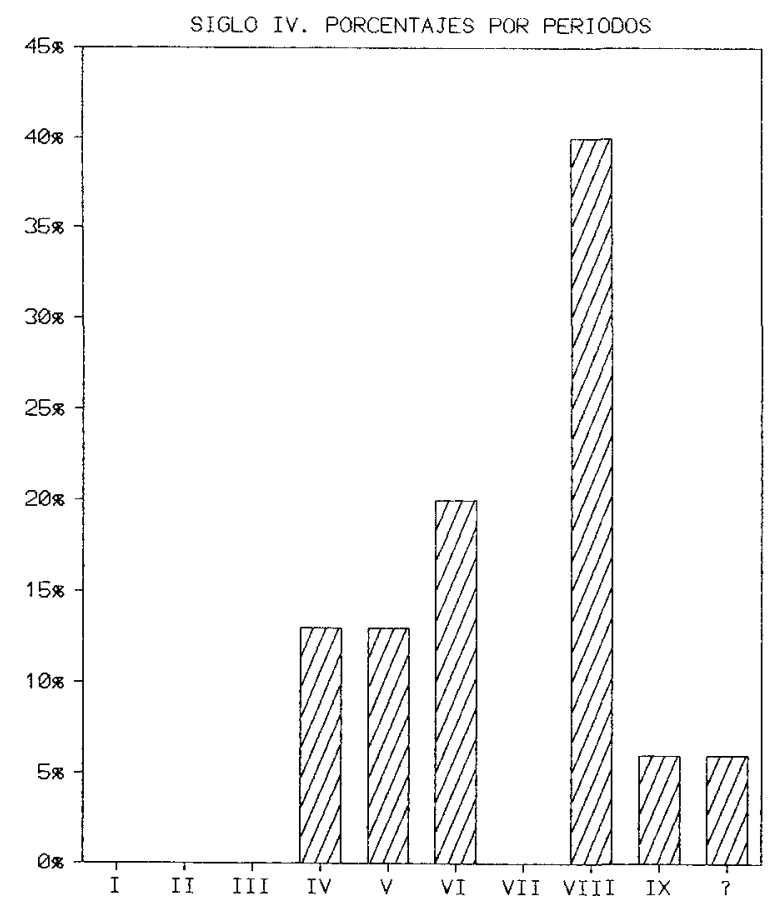

Fig. 3. - Distribución por periodos de las monedas del siglo IV en La Almadrava. I: 307-318; II: 318-324; III: 324-330; IV: 330-335; V: 335-341; VI: 341-348; VII: 348-350; VIII: 351-364; IX: 364-408; ?: atribución imprecisable siglos IV-V.

Aunque en términos globales la circulación media no supera las 0'15 monedas/año, si consideramos que la pieza más antigua es de la década del 330 y la más moderna no supera seguramente el 392, la media se eleva hasta 0'23.

No tiene demasiado sentido cuantificar un conjunto tan reducido. Sin embargo, La Almadrava coincide con el resto de los enclaves del occidente latino en la considerable presencia de las monedas de Constancio II y Constante ( 7 piezas), el $46^{\prime} 6 \%$ del siglo IV, resultado de las abultadas emisiones de todas las cecas en estos años (Fig. 3). 
ALMADRAVA. SIGLO IV

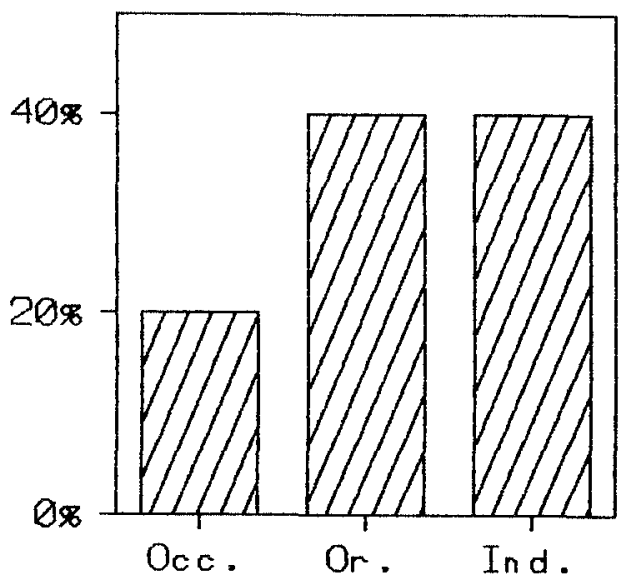

PROCEDENCIA DE LAS MONEDAS

Fig. 4.- Procedencia regional de las monedas del siglo IV en La Almadrava.

Por tipos, no faltan en La Almadrava los característicos reversos de victoriae dd auggq $n n$ y vot $x x$ mult $x x x$ de los años 347-348, el spes reipublice de 355-361, o los frecuentes fel temp reparatio que definen por antonomasia el monetario occidental de Constancio II. En el conjunto destacan también una pequeña pieza de las series de Populus Romanus de Constantinopla ( ${ }^{\circ}$ 63) y un AE 2 corto de Magnencio con el conocido reverso de dos Victorias sosteniendo escudo $\left(\mathrm{n}^{\circ} 68\right)$.

En el reducido análisis de cecas que del conjunto se puede hacer (Fig. 4), contra lo habitual en el área levantina, el $20 \%$ de las piezas proceden de ceca occidental (exclusivamente Roma) y el $4 \%$ de cecas orientales (Heraclea, Constantinopla, Cyzico y desconocida); restan 6 piezas de procedencia desconocida entre las que, de emplear criterios observados en otros lugares, la mayor parte debe ser occidental (Tabla 5).

La última moneda catalogable del monetario del siglo IV en La Almadrava es un AE 3 de ceca desconocida, acuñado a nombre de un Valentiniano; el reverso no permite datar la pieza, pero sí situarla como muy tarde hacia el 392 d.C., fecha terminal en el registro numismático del yacimiento.

\subsection{El medallón adrianeo}

La pieza más excepcional del conjunto de hallazgos de La Almadrava es el medallón de Adriano ( ${ }^{\circ}$ 15 ), hallado desgraciadamente en un contexto poco significativo. Aunque la acuñación de medallones es característica de los últimos años del reinado

TABLA 5

\begin{tabular}{|c|c|c|c|c|c|c|c|c|c|c|c|c|c|c|c|}
\hline \multicolumn{2}{|c|}{ PERÍ́ODO } & I & II & III & IV & $\mathbf{v}$ & VI & VII & VIII & IX-X & & & & & \\
\hline & & \multirow{2}{*}{$\begin{array}{l}307 \\
318\end{array}$} & \multirow{2}{*}{$\begin{array}{l}318 \\
324\end{array}$} & \multirow{2}{*}{$\begin{array}{l}324 \\
330\end{array}$} & \multirow{2}{*}{$\begin{array}{l}330 \\
335\end{array}$} & \multirow{2}{*}{$\begin{array}{l}335 \\
341\end{array}$} & \multirow{2}{*}{$\begin{array}{l}341 \\
348\end{array}$} & \multirow{2}{*}{$\begin{array}{l}348 \\
350\end{array}$} & \multirow{2}{*}{$\begin{array}{l}351 \\
364\end{array}$} & \multirow{2}{*}{$\begin{array}{l}364 \\
392\end{array}$} & \multirow[t]{2}{*}{$?$} & \multicolumn{2}{|c|}{ Total } & \multicolumn{2}{|c|}{ Total } \\
\hline & & & & & & & & & & & & N. & & \multicolumn{2}{|c|}{ N. ${ }^{o} \quad \%$} \\
\hline Occ. & Rom & & & & & 2 & 1 & & & & & 3 & 20 & 3 & 20 \\
\hline \multirow{4}{*}{ Or. } & Her & & & & 1 & & & & & & & 1 & 6,6 & \multirow{4}{*}{6} & \multirow{4}{*}{40} \\
\hline & $\mathrm{Cp}$ & & & & 1 & & & & & & & 1 & 6,6 & & \\
\hline & $\mathrm{Cyz}$ & & & & & & 1 & & 2 & & & 3 & 20 & & \\
\hline & Or? & & & & & & 1 & & & & & 1 & 6,6 & & \\
\hline \multicolumn{2}{|l|}{$?$} & & & & & & & & 4 & 1 & 1 & 6 & 40 & 6 & 40 \\
\hline \multirow{2}{*}{ Total } & N. ${ }^{o}$ & & & & 2 & 2 & 3 & & 6 & 1 & 1 & 15 & & 15 & \\
\hline & $\%$ & & & & 13,3 & 13,3 & 20 & & 40 & 6,6 & 6,6 & & 100 & & 100 \\
\hline
\end{tabular}

Tabla 5._ Procedencia por cecas de las monedas del siglo IV en La Almadrava. 
de Adriano (KENT 1978, 31), la mención del segundo consulado del Emperador en el reverso no deja no deja lugar a dudas sobre su datación: se trata de un ejemplar del año 118.

En el anverso de la pieza figura un característico retrato adrianeo de sus primeros años, y la leyenda es la habitual del momento. En el reverso aparece la leyenda P M TR P COS II y una escena de loba con gemelos, rara en la amonedación de Adriano - prácticamente exclusiva de cuadrantes- que no falta, sin embargo, en este tipo de medallas.

$\mathrm{Si}$ los medallones se diferencian de las series monetales por la ausencia de la fórmula S C (TOYNBEE 1944, passim; SUTHERLAND 1974, 201), el ejemplar de La Almadrava constituye una excepción, ya que la fórmula citada figura en el exergo. Las características y las leyendas del medallón coinciden, incluso en las medidas, con las de otro ejemplar descrito por Cohen de la venta de la colección Laborde (COHEN 1955, $\mathrm{n}^{\circ}$ 1055) cuya lectura han puesto en duda trabajos posteriores (MATTINGLY y SYDENHAM 1972, 409, n. 559).

\section{NUMISMÁTICA Y EVIDENCIA ARQUEOLÓGICA}

\subsection{Los hallazgos y la actividad del complejo artesanal.}

Las setenta y cinco piezas que presentamos en este estudio, pese a lo limitado de su número, constituyen un conjunto importante en el marco de los hallazgos monetarios en villae rústicas de la Tarraconense mediterránea. Su importancia viene dada, además, por su condición de piezas procedentes de excavación, lo que implica que se dispone de documentación acerca de su correspondientes contextos arqueológicos.

Con excepción de una pieza, el resto se ajusta sin dificultad con el marco cronológico de uso del enclave, sin datos posteriores al siglo V d.C. Desde la perspectiva de la distribución temporal, los más significativos conjuntos de piezas coinciden con la datación of recida por las principales fases constructivas y de actividad tanto en el complejo alfarero como en la zona residencial.
La ausencia de piezas relacionables con la primera fase se explica por la ausencia de estructuras arquitectónicas de este período; pero no ocurre lo mismo con la segunda fase (siglo II) o con la tercera: el volumen de monedas perteneciente al tercer cuarto del siglo III d.C., con independencia de su relación con fenómenos de aumento del volumen de las emisiones o de otros derivados de la dinámica de la circulación monetaria, debe valorarse en función de la presencia de muchas de estas piezas en unidades estratigráficas correspondientes al momento de abandono de la actividad artesanal, en torno a los años 275-285 d.C. En efecto, el buen estado de conservación de algunas de estas unidades, no afectadas por remodelaciones posteriores, ha permitido la supervivencia de las piezas. Del mismo modo, las monedas de cronología posterior, disponen ya de contextos arqueológicos muy amplios y poco definidos, siendo su hallazgo frecuente en unidades muy superficiales y con escasa fiabilidad estratigráfica en razón del arrasamiento de las estructuras tardías.

Sin olvidar las limitaciones de la datación arqueológica a partir de la evidencia numismática, principalmente debidas a los largos períodos de circulación de las piezas y siempre con valor post quem, la evidencia de algunos contextos de La Almadrava sugiere no exagerar las precauciones. Si nos atenemos a las unidades U.E. 86-3-18 y U.E. 86-3-53, con cinco monedas cada una, podemos comprobar que las fechas de emisión de las piezas, en cuatro de cada cinco casos, oscila en un margen máximo de 20 años, mientras el quinto ejemplar corresponde a piezas de circulación dilatada, residuales o intrusiones, dependiendo del contexto.

En la mayoría de las unidades estratigráficas fiables (por ejemplo U.E. 86-3-63 y U.E. 86-3-67) la fecha de emisión de las monedas coincide con el marco cronológico, relativo aunque bastante específico, que ofrecen los materiales cerámicos y otros elementos del registro material.

Otro aspecto a destacar es la relación entre el estado de conservación de las piezas y su procedencia estratigráfica. Del inventario expuesto con anterioridad se deduce que un altísimo porcentaje de monedas en mal estado o de difícil clasificación proceden de los estratos más superficiales -U.E. 1 y U.E. 2-, notablemente alterados, y que las piezas mejor conservadas se relacionan con los contextos arqueológicos estratigráficamente más fiables. 
TABLA 6

\begin{tabular}{|c|c|c|c|c|c|c|c|}
\hline N. ${ }^{\circ}$ & $\begin{array}{l}\text { N. }{ }^{\circ} \text { INV. } \\
\text { MUSEO }\end{array}$ & $\begin{array}{c}\text { FECHA DE } \\
\text { HALLAZGO }\end{array}$ & $\begin{array}{c}\text { CONTEXTO } \\
\text { (SIGNATURA }+ \\
\text { UNIDAD) }\end{array}$ & N. ${ }^{\circ}$ & $\begin{array}{l}\text { N. }{ }^{\circ} \text { INV. } \\
\text { MUSEO }\end{array}$ & $\begin{array}{c}\text { FECHA DE } \\
\text { HALLAZGO }\end{array}$ & $\begin{array}{c}\text { CONTEXTO } \\
\text { (SIGNATURA } \\
\text { UNIDAD) }\end{array}$ \\
\hline 1 & 47 & $7-89$ & $89-7-1$ & 38 & 39 & $8-87$ & $87-6-157$ \\
\hline 2 & 29 & $10-87$ & $88-3-1$ & 39 & 50 & $7-89$ & $89-7-1$ \\
\hline 3 & 26 & $5-87$ & $87-6-1$ & 40 & 63 & $4-90$ & $90-7-1$ \\
\hline 4 & 71 & $7-90$ & $90-15-1$ & 41 & 51 & $7-89$ & $89-7-1$ \\
\hline 5 & 72 & $7-90$ & $90-15-1$ & 42 & 17 & $11-86$ & $86-3-53$ \\
\hline 6 & 64 & $5-90$ & $90-7-1$ & 43 & 35 & $10-87$ & $88-3-2$ \\
\hline 7 & 7 & $6-86$ & $86-2-2$ & 44 & 27 & $7-87$ & $87-6-1$ \\
\hline 8 & 22 & $10-86$ & $86-3-118$ & 45 & 11 & $11-86$ & $86-3-18$ \\
\hline 9 & 32 & $11-87$ & $88-3-1$ & 46 & 31 & $10-87$ & $88-3-1$ \\
\hline 10 & 5 & $8-86$ & $86-3-1$ & 47 & 21 & $11-86$ & $86-3-67$ \\
\hline 11 & 67 & $7-90$ & $90-15-1$ & 48 & 10 & $11-86$ & $86-3-18$ \\
\hline 12 & 3 & $8-86$ & $86-3-1$ & 49 & 104 & $7-86$ & $85-2-11$ \\
\hline 13 & 56 & $8-88$ & $88-3-2$ & & & & (sector B) \\
\hline 14 & 57 & $8-88$ & $88-3-2$ & 50 & 53 & $6-89$ & $89-7-2$ \\
\hline 15 & 43 & $9-88$ & 88-3-1 & 51 & 16 & $11-86$ & $86-3-53$ \\
\hline 16 & 15 & $11-86$ & $86-3-53$ & 52 & 9 & $11-86$ & $86-3-18$ \\
\hline 17 & 54 & $10-89$ & $89-7-294$ & 53 & 28 & $10-87$ & 88-3-1 \\
\hline 18 & 19 & $11-86$ & $86-3-65$ & 54 & 45 & 7-89 & $89-7-2$ \\
\hline \multirow[t]{2}{*}{19} & 62 & $7-88$ & $88-3-2$ & 55 & 24 & $7-87$ & $87-6-1$ \\
\hline & & & (442) & 56 & 58 & $8-88$ & $88-3-2$ \\
\hline 20 & 1 & $5-85$ & $86-3-1$ & 57 & 46 & $7-89$ & $89-7-2$ \\
\hline 21 & 20 & $11-86$ & $86-3-66$ & 58 & 102 & $7-86$ & $85-2-1$ \\
\hline \multirow[t]{2}{*}{22} & 103 & $7-86$ & $85-2-11$ & 59 & 37 & $8-87$ & $87-6-149$ \\
\hline & & & (sector B) & 60 & 38 & $7-87$ & $87-6-149$ \\
\hline 23 & 4 & $8-86$ & $86-3-1$ & 61 & 13 & $11-86$ & $86-3-18$ \\
\hline 24 & 14 & $11-86$ & $86-3-53$ & 62 & 34 & $10-87$ & $87-6-2$ \\
\hline 25 & 68 & $7-90$ & $90-15-1$ & 63 & 2 & $5-85$ & $86-3-1$ \\
\hline 26 & 69 & $9-90$ & $90-15-1$ & 64 & 52 & $6-89$ & $89-7-2$ \\
\hline 27 & 65 & $5-90$ & $90-7-1$ & 65 & 30 & $10-87$ & $88-3-1$ \\
\hline 28 & 6 & $9-86$ & $86-3-1$ & 66 & 23 & $10-86$ & $86-3-119$ \\
\hline 29 & 8 & $12-86$ & $86-3-44$ & 67 & 36 & $10-87$ & $88-3-2$ \\
\hline 30 & 25 & $6-87$ & $87-6-1$ & 68 & 49 & $7-89$ & $89-7-1$ \\
\hline 31 & 40 & $12-87$ & $88-3-224$ & 69 & 59 & $8-88$ & $88-3-2$ \\
\hline 32 & 12 & $11-86$ & $86-3-18$ & 70 & 66 & $7-90$ & $90-15-1$ \\
\hline 33 & 41 & $12-87$ & $88-3-236$ & 71 & 61 & $3-88$ & $88-3-268$ \\
\hline 34 & 44 & $7-89$ & $89-7-2$ & 72 & 60 & $9-88$ & $88-3-436$ \\
\hline 35 & 55 & $7-88$ & $88-3-2$ & 73 & 33 & $10-87$ & $88-3-2$ \\
\hline 36 & 18 & $11-86$ & $86-3-53$ & 74 & 48 & $7-89$ & $89-7-1$ \\
\hline 37 & 70 & $8-90$ & $90-15-1$ & 75 & 101 & 4-85 & $85-2-1$ \\
\hline
\end{tabular}

Tabla 6.- Correspondencia entre los números del catálogo y los de inventario monetal del Museo Arqueológico de Denia. 


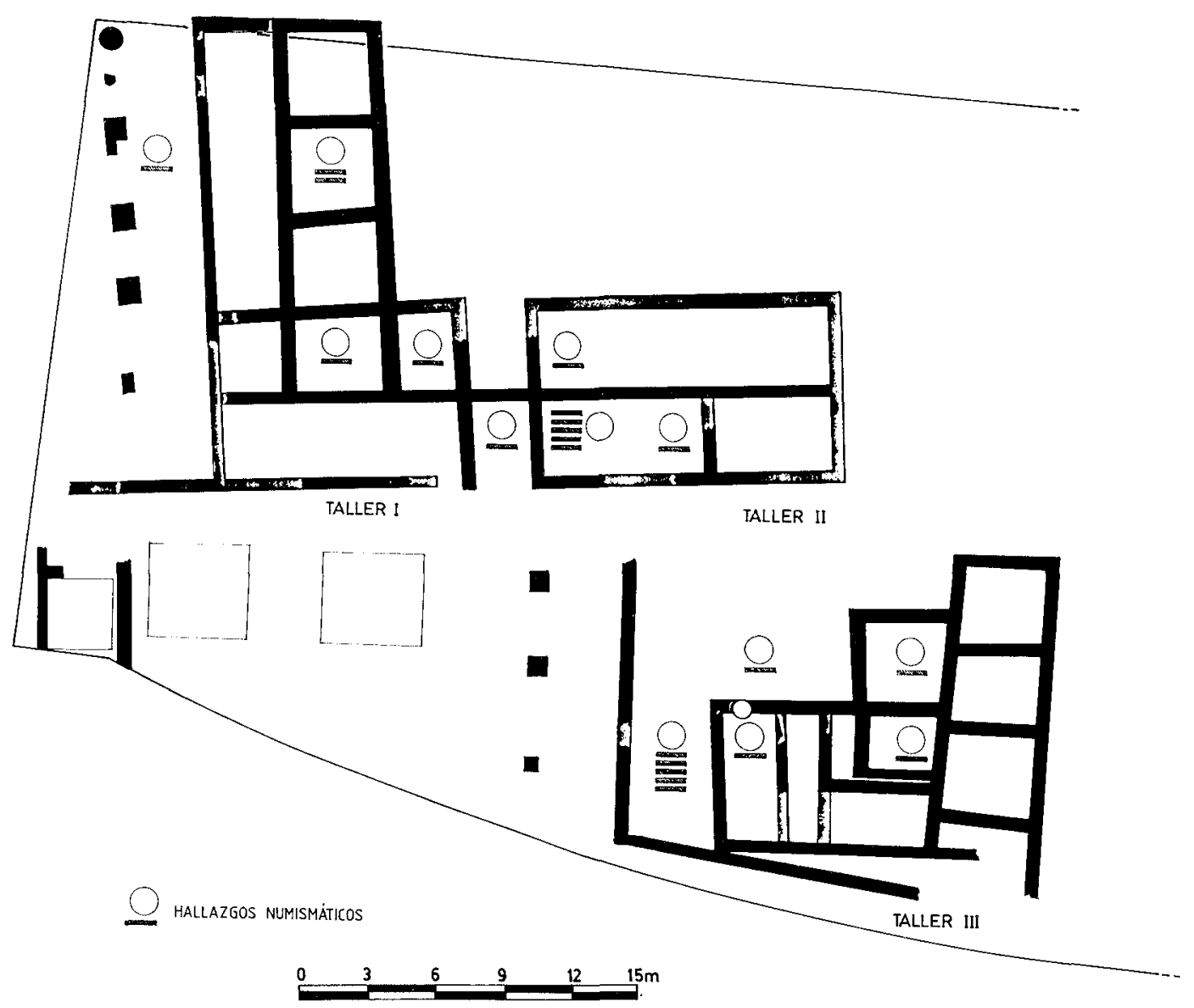

Fig. 5. - Planta del sector alfarero con situación de talleres individualizados y dispersión de hallazgos numismáticos en los distintos ambientes.

\subsection{Hallazgos numismáticos y contexto arqueológico}

Tras la presentación sinóptica de las grandes líneas que definen el funcionamiento del alfar, y visto el ritmo de la circulación monetaria, resta analizar ahora los contextos arqueológicos en que se hallan insertos los hallazgos monetarios de $\mathrm{La} \mathrm{Al}$ madrava (Tabla 6).

Si partimos del hecho de que la excavación en el complejo de La Almadrava ha proporcionado tan sólo setenta y cinco monedas, y de que muchas de ellas proceden de unidades genéricas en niveles superficiales, la singularidad de algunos contextos rentabiliza cualquier esfuerzo de análisis. La trascendencia de la relación entre hallazgos numismáticos y arqueológicos aumenta en el caso de este tipo de instalaciones industriales, en donde el registro adolece con frecuencia de materiales de datación precisa y en donde, pese a sus limitaciones, la moneda se convierte en el elemento principal de fijación cronológica (Fig. 5).

La unidad estratigráfica U.E. 1, estrato más superficial y extensible a todo el yacimiento, sensiblemente alterado por el uso continuado del suelo de aprovechamiento agrícola, contiene el $45 \%$ de las monedas -34 ejemplares-, con una distribución cronológica equiparable a la general del conjunto numismático. El porcentaje de piezas de atribución indeterminada es elevado, debido a su mal estado de conservación.

La unidad estratigráfica U.E. 2 subyace a la anterior y tiene una gran similitud, con la particularidad de estar menos alterada y cubrir o asentarse so- 
bre unidades estratigráficas fiables. La distribución cronológica de las piezas es distinta, al no contener monedas de carácter residual y predominar las pertenecientes a las últimas etapas de la secuencia del alfar, debido a su papel de estrato regularizador de unidades subyacentes. Contiene el $20 \%$ de las monedas - 15 piezas-. El mapa de distribución espacial de los hallazgos en estas unidades no presenta particularidad alguna. Se trata de hallazgos puntuales y su situación es puramente aleatoria, con una dispersión irregular que afecta a los distintos sectores de excavación.

\section{ANALISIS NUMISMATICO DEL SECTOR ALFARERO}

\subsection{EI Taller I}

En el Taller I, pese a constituir el mejor exponente y un modelo dentro del complejo alfarero en lo que se refiere a la función de las estructuras y la técnica edilicia, los hallazgos numismáticos apenas revisten interés, debido a su dispersión y escasa concentración. De ahí que omitamos la descripción de los contextos, sobre los que incidiremos al tratar el Taller II. Los hallazgos de monedas afectan a cuatro de los diez ambientes que conforman el Taller I. Las piezas halladas en ambientes que, según la arqueología, están relacionados con el proceso de producción cerámica, tienen carácter resdiual o constituyen intrusiones resultado de la contaminación de sus unidades respectivas. Este es el caso de las unidades U.E. 118 y 119 respectivamente. Más acorde y, en principio, compatible con su contexto es el caso de la unidad U.E. 157, integrada dentro de una galería porticada en donde se procede a la preparación de la arcilla.

La unidad U.E. 149 es el estrato de colmatación de un pequeño depósito de planta circular y base convexa excavado en el subsuelo -U.E. 148--, situado en el interior de una pequeña estancia de planta cuadrangular y colindante con una estructura que se interpreta como banco de cocina. Entre claros desechos orgánicos (malacología terrestre y fauna) se hallaron dos monedas. Curiosamente, este hallazgo se circunscribe a un ambiente del taller destinado a hábitat. La cronología de las monedas, 303 d.C., constituye la única evidencia sobre la con- tinuidad de uso de uno de los ambientes del complejo alfarero con posterioridad al 275 d.C.

Los hallazgos de este taller son los siguientes (6):

- U.E. 118: $\mathrm{n}^{\circ}$ 8: 86 d.C.

- U.E. $119: \mathrm{n}^{\circ}$ 66: 347-348 d.C.

- U.E. 149: $n^{\circ}$ 59: 303 d.C. $n^{\circ}$ 60: 303 d.C.

- U.E. 157: $\mathrm{n}^{\circ}$ 38: 266 d.C.

\subsection{El Taller II}

El Taller II es, sin duda, el edificio del complejo alfarero con un mayor número de hallazgos numismáticos en unidades estratigráficas con un alto grado de fiabilidad. Ofrece un total de siete piezas, distribuidas en tres unidades: dos estratos de preparación de un pavimento -U.E. 65 y 66-, íntimamente ligados entre sí, y un estrato que documenta el momento de ocupación y posterior abandono del edificio - U.E. 53-. Al tratarse de los contextos de mayor interés en relación con la presencia de monedas, describimos de modo particular los rasgos definitorios de estas unidades.

EI Taller II (Fig. 6) es un edificio de planta rectangular cuyos muros perimetrales están construidos con mampostería irregular careada y sin unión (opus incertum) sirviendo de zócalo, mientras el resto del muro lo constituye un paramento de barro $o$ arcilla. Presenta compartimentaciones en el interior, con muros de mayor amplitud, de los que se conserva tan sólo una hilada de cantos rodados - gravas-, que se completaría con paramento de barro. Se trata de un edificio construido ex novo específicamente para ser destinado a la actividad del alfar.

\subsubsection{Unidad estratigráfica 86-3-65}

Definición. Estrato de relleno y nivelación del terreno, asentado sobre el suelo geológico. Presenta como componentes geológicos arcillas de color marrón, con abundantes manchas de color rojizo, producto de la descomposición de adobes.

(6) Los números de las monedas corresponden a los del inventario. En la tabla 6 puede verse la correspondencia con los números de registro del Museo Arqueológico de Denia. 


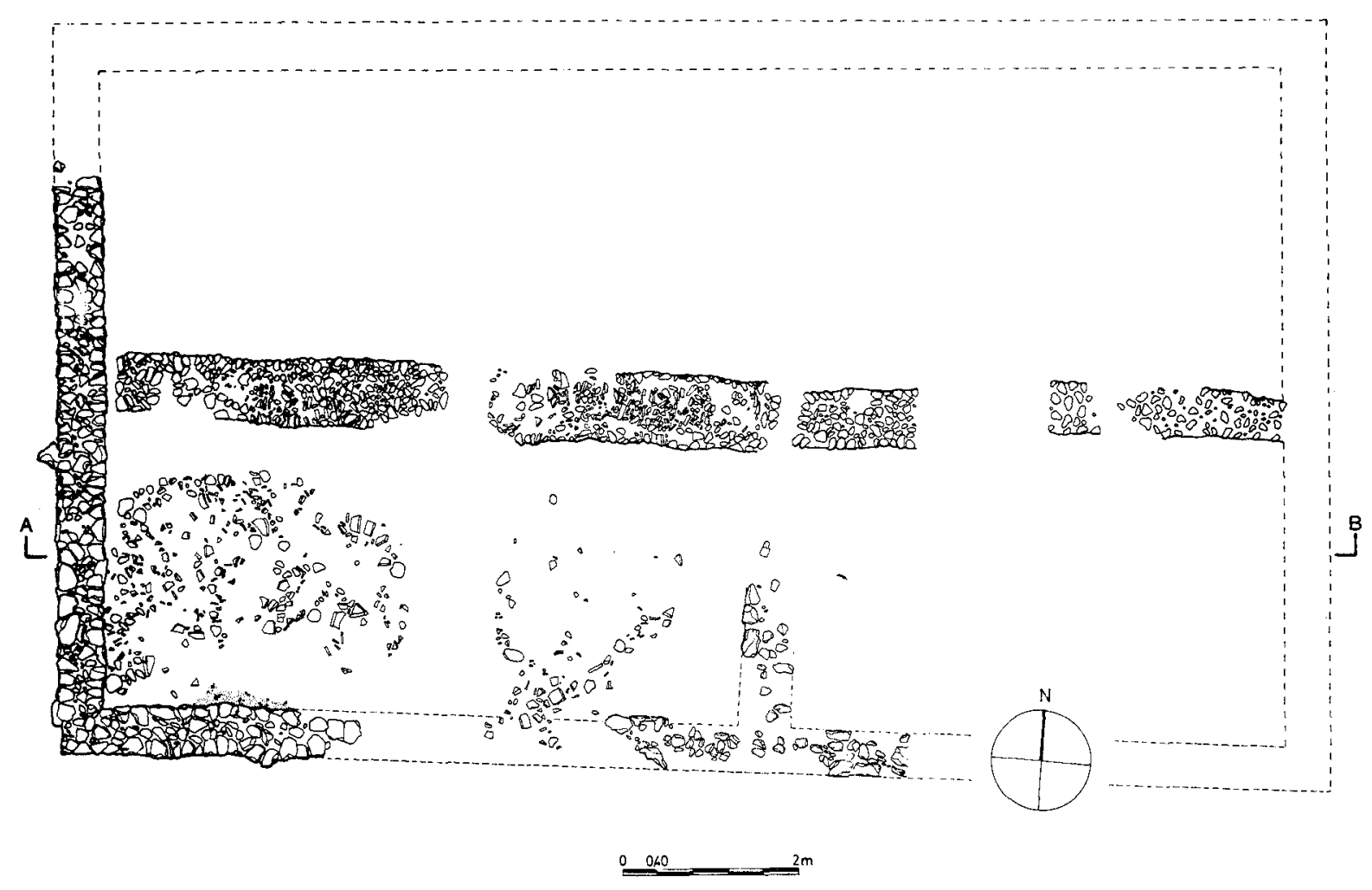

TALLER $\|$

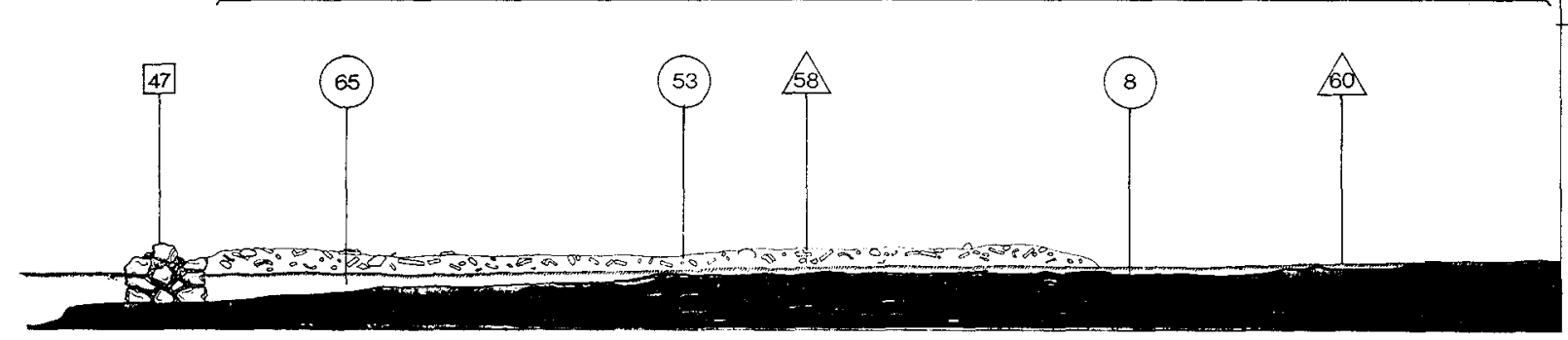

SECCIÓ A.B

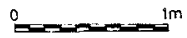

Fig. 6.- Taller II. Situación de unidades estatrigráficas y contextos arqueológicos con hallazgos numismáticos. 4-1: Planta: 4-2: Sección A-B.

Interpretación. Estrato de preparación del pavimento de uno de los ambientes del taller II. Su formación se asocia a la tercera fase de la secuencia del alfar.

Registro arqueológico. Destaca la abundancia de materiales de contrucción fragmentarios, ánforas (con amplio predominio de Dressel 30) y cerámicas comunes, producción habitual del alfar. La escasez de cerámicas finas contrasta con la presencia de diversos fragmentos de placas y molduras de mármol, así como restos de fauna. Es el contexto de un fragmento de inscripción funeraria (ABASCAL - GIS- 
BERT 1990, $\mathrm{n}^{\circ}$ I.2). Ello sugiere que la formación de este estrato de nivelación integra materiales, al parecer, procedentes de un área de hábitat que posiblemente es el sector residencial adyacente. Los materiales más representados nos sitúan en el segundo tercio del siglo II d.C., y el pavimento que sella esta unidad se situaría hacia el 230 d.C. Coincide con la datación relativa de la inscripción.

Hallazgos numismáticos. Moneda $\mathrm{n}^{\circ}$ 18: 145 d.C.

\subsubsection{Unidad estratigráfica 86-3-66}

Definición, interpretación y registro similar a la anterior.

Hallazgos numismáticos. Moneda $\mathrm{n}^{\circ} 21$ : 164-169.

\subsubsection{Unidad estratigráfica 86-3-53}

Definición. Estrato de colmatación, de desarrollo irregular, que se asienta sobre un pavimento o suelo de tierra apisonada -U.E. 58-. Su componente geológico es tierra arcillosa de color marrón oscuro, con abundantes cantos rodados - gravasy mampuestos calcáreos procedentes del derrumbe de los muros. Presenta un alto componente de tegulae e imbrices fragmentarios, así como un importante registro de cerámicas.

Interpretación. Esta unidad documenta conjuntamente la caída de la cubierta, derrumbe de los muros $\mathrm{y}$, en definitiva, el abandono y destrucción del edificio, junto a los niveles de ocupación del ambiente en cuestión. La densidad de tegulae confirma la tipología de la cubierta del edificio y el registro arqueológico es el característico de la producción del alfar y confirma el uso artesanal del inmueble.

Registro arqueológico. Destaca la escasa presencia de cerámicas finas, así como el carácter residual de las mismas, frente a la abundancia de ánforas, en las que predomina el tipo Dressel 30; aparecen también cerámicas comunes de mesa y conserva. $\mathrm{La}$ vajilla africana de cocina y mesa sitúa el contexto en el segundo tercio del siglo III d.C.

Hallazgos numismáticos:

- Moneda $\mathrm{n}^{\circ}$ 24: Segunda mitad del siglo II d.C.

- Moneda $n^{\circ}$ 16: 132-134 d.C.

- Moneda $\mathrm{n}^{\circ}$ 51: c. 270 d.C.

- Moneda $n^{\circ}$ 42: 268 d.C.

- Moneda $n^{\circ}$ 36: 266 d.C.
En esta unidad, la numismática ofrece una información excepcional para plantear el final de la producción del alfar y el abandono del sector artesanal de la villa.

\subsubsection{Unidad estratigráfica 83-3-67}

Está situada entre los talleres I y II y comparte la definición e interpretación con las unidades U.E. 65 y 66.

Hallazgos numismáticos:

- Moneda $n^{\circ}$ 47: 269-270 d.C.

\subsection{EI Taller III}

El taller III presenta algunos hallazgos numismáticos en contextos de similares características y sincrónicos con los descritos en el taller II.

\subsubsection{Unidades estratigráficas 88-3-224 y 88-3-236}

Las unidades estratigráficas U.E. 224 y 236 se definen como estratos de acumulación que descansan sobre pavimentos, y se interpretan como niveles de ocupación y abandono del edificio durante el tercer cuarto del siglo III d.C.

Los hallazgos numismáticos de estas unidades son los siguientes:

- U.E. 224: $\mathrm{n}^{\circ}$ 31: 238-239 d.C.

- U.E. 236: $n^{\circ}$ 33: 244-247 d.C.

\subsubsection{Unidad estratigráfica 88-3-44}

La unidad estratigráfica U.E. 41 es un pozo excavado en el suelo geológico. Su construcción rompe uno de los muros perimetrales del taller III, lo que confirma su cronología bajo-imperial. La unidad U.E. 44 es un estrata de colmatación de este pozo.

El único hallazgo numismático de esta unidad es el siguiente:

$$
\text { - Moneda } \mathrm{n}^{\circ} \text { 29: } 228 \text { d.C. }
$$

\subsubsection{Unidad estratigráfica 89-7-294}

La unidad U.E. 89-7-294 es un estrato de relleno y se interpreta como de nivelación y preparación del pavimento o suelo de tierra apisonada U.E. 327. 
El único hallazgo numismático de esta unidad es el siguiente:

- Moneda $n^{\circ} 17: 139$ d.C.

\subsubsection{Unidad estratigráfica 86-3-18}

Junto al muro perimetral occidental del taller III se localiza la unidad estratigráfica U.E. 18, al exterior del mismo, en un área interpretada como de uso comunal. Es una deposición que se forma en el momento final de la actividad del sector artesanal, sincrónicamente a una serie de unidades que definen el abandono de los talleres.

Definición. Bolsada con un componente de arcilla negruzca con aporte de cenizas, abundantes gravas de pequeño módulo y materiales orgánicos. Presenta un alto porcentaje de cerámicas, con mezcla de un registro claramente definitorio de la producción del alfar, mezclado con otro característico de un ambiente de hábitat. Esta duplicidad señala su carácter de aporte de escombros procedente de un ambiente de hábitat del sector residencial.

Interpretación. Deposición de escombros, con las peculiaridades descritas.

Registro arqueológico. Entre las cerámicas finas abundan las formas de vajilla de mesa y cocina africanas, encuadrables en la primera mitad del siglo III d.C. La presencia de cerámicas de cocina con marcas de uso, de objetos diversos de bronce y hueso, así como de abundante fauna, confirma la procedencia ya descrita de los materiales. En cuanto al registro propio del alfar, presenta éste un amplio repertorio de cerámica común, y entre las ánforas predomina ampliamente la Dressel 30.

Hallazgos numismáticos:

- Moneda $n^{\circ}$ 52: c. 270 d.C.

- Moneda $n^{\circ}$ 48: 269-270 d.C.

- Moneda $n^{\circ}$ 45: 269 d.C.

- Moneda $\mathrm{n}^{\circ}$ 32: 247-249 d.C.

- Moneda $n^{\circ}$ 61: 335-337 d.C. (posible intrusión)

\section{ANÁLISIS NUMISMÁTICO DEL SECTOR RESIDENCIAL}

\subsection{Unidades estratigráficas 436 y 268}

En cuanto a los contextos arqueológicos con hallazgos numismáticos pertenecientes al sector resi- dencial de la villa, destaca la ausencia de estos en el edificio termal. El hecho de que este sector se halle aún en proceso de excavación determina que los contextos de que disponemos sean bolsadas o deposiciones, sin relación directa con estructuras arquitectónicas, lo que dificulta su interpretación. Este es el caso de las unidades estratigráficas U.E. 436 y 268 , con materiales arqueológicos pertenecientes a la etapa bajoimperial de la villa. Los hallazgos numismáticos de estas unidades son los siguientes:

$$
\text { - U.E. 436: } \mathrm{n}^{\circ} \text { 72: 354-361 d.C. }
$$

\subsection{Unidad estratigráfica 88-3-2 (442)}

El contexto de la unidad estratigráfica 88-3-2 (442) es de cronología anterior y se relaciona con una de las fases del edificio de las termas. Se trata de una bolsada con abundantes cenizas, situada al norte del mismo. Presenta abundantes materiales de construcción, así como un conjunto de vajilla africana que sitúa la formación de este estrato en la mitad del siglo II d.C. Entre los materiales destaca la única pieza de plata, un denario de Marco Aurelio.

El único hallazgo numismático de esta unidad es el siguiente:

$$
\text { - Moneda } \mathrm{n}^{\circ} \text { 19: } 168 \text { d.C. }
$$

\subsection{Unidad estratigráfica 88-3-1}

Pertenece esta unidad al sector residencial, aún en curso de excavación, en las proximidades del conjunto termal; se trata de una unidad superficial, sin conteto arqueológico específico. A esta unidad pertenece el excepcional medallón de Adriano, $\mathrm{n}^{\circ}$. 15, datado el año 118 d.C. (7).

\section{CONCLUSIONES}

$\mathrm{El}$ análisis de los hallazgos numismáticos en $\mathrm{La}$ Almadrava revela un período de uso del lugar des-

(7) Omitimos la descripción de los contextos del sector B de la Almadrava al no plantear cuestiones de interés. 
de mediados del siglo I a.C. hasta fines del siglo IV / comienzos del V d.C., en consonancia con la interpretación de los datos arqueológicos. Los testimonios de la excavación, corroborados por los hallazgos de monedas, determinan el fin de la actividad del alfar entre los años 275-285 d.C., quedando el enclave como lugar de residencia durante el siglo IV.

Durante la fase de actividad el asentamiento sigue pautas de circulación monetaria similares a las de otros enclaves de la costa mediterránea, aunque apartándose del modelo en la menor incidencia de las cecas hispanorromanas de la primera mitad del siglo I d.C. y en una media de circulación anual inferior a las de otros lugares, aunque la relación de unos períodos con otros es proporcional a los patrones generales.

La interpretación conjunta de las unidades arqueológicas y sus hallazgos monetarios muestra, en general, una correspondencia entre unidades homogéneas y monedas en buen estado de conservación, de lo que puede deducirse que las piezas halladas se extraviaron probablemente cuando aún constituían numerario circulante; ello aumenta su importancia en la datación de las estructuras y confirma que el deterioro acusado de algunos ejemplares es posterior a su etapa de uso, al coincidir además su ubicación con unidades arqueológicas sin homogeneidad cronológica.

\section{BIBLIOGRAFIA}

ABASCAL, J. M. 1989. La circulación monetaria del Portus Ilicitanus. Valencia.

ABASCAL, J.M. y GISBERT, J.A. 1990. «Epigrafía romana de la villa de l'Almadrava (Setla - Mirarosa - Miraflor). Apéndice: «Nuevas aportaciones a la epigrafía de Dianium», en III Congres d'Estudis Comarcals, Institut d'Estudis Comarcals Marina Alta. Novembre 1990 (en prensa).

ARANEGUI, C. y GISBERT, J. A. 1990. «El alfar romano de l'Almadrava (Setla - Mirarosa - Miraflor). La producción de ánforas Dressel 30 en el territorium de Dianium», en III Congres d'Estudis Comarcals, Institut d'Estudis Comarcals Marina Alta. Novembre 1990 (en prensa).

ARROYO, R. 1980. «Vespasiano y su proyección monetaria en las tierras valencianas», Saguntum 15, 191-206.

- 1982. El Numario de la Universidad de Valencia. Valencia.

- 1987-88. «Estudio numismático del yacimiento tardorromano del Albir (Altea-Alacant)», Acta Numismática 17-18, 153-172.
BALIL, A. 1958. «Circulación monetaria en Hispania en el Imperio Romano", Numisma 35, 25-29.

BOON, G.C. 1978. "Monnaies fausses de l'époque impériale», en Les devaluations à Rome. Coll. Ecole Franç. Rome 37. Roma, 99-106.

BOST, J. P. y PEREIRA, I. 1974. «Les monnaies d'imitation de Claude I trouvées sur le site de Conimbriga (Portugal)», I Congr. Nac. Numism. (Zaragoza 1973). Zaragoza, $167-181$.

BRUUN, P. A. 1984. The roman imperial coinage VII: Constantine and Licinius. $A D$ 313-337. London (rptd. de la edición de 1966).

CALLU, J. P. 1969. La politique monétaire des empereurs romains de 238 à 311 . Paris.

- 1974. «Remarques sur le trésor de Thamusida III: les Divo Claudio en Afrique du nord. Note additionnelle de Pierre Salama», MEFRA 86.1, 523-540.

CAMPO, M. 1974. «El problema de las monedas de imitación de Claudio I en Hispania», Acta Numismática 4, 155-163.

- 1979. «Circulación monetaria en Menorca», I Symposium numismático de Barcelona, vol. I, 97 ss.

CARSON, R.A.G. y KENT, J.P.C. 1978, «Bronze roman imperial coinage of the later Empire A.D. 346-498», en Late roman bronze coinage A.D. 324-498 (part II). Londres (rptd.).

CASEY, P. J. 1982. «The Coins», en Corbridge, Excavations of the Roman fort and town, 1947-80. English Heritage, archaeological report 8; Historic Building - Monuments from England, pp. 142-158.

CENTENO, R.M.S. 1981-82. «A circulaçao dos Divo Claudio na Península Ibérica: notas sobre um tesouro do concelho de Amarante», Portugalia, N.S., 2-3, 122-129.

COHEN, H. 1955. Description historique des monnaies frappées sous l'Empire romain communement appelées medailles imperiales. Graz.

FERNÁNDEZ, A. y GISBERT, J. A. 1990. «Investigaciones arqueológicas subacuáticas en las costas de Denia 1985-1989», en III Congres d'Estudis Comarcals, Institut d'Estudis Comarcals Marina Alta. Novembre 1990 (en prensa).

GIARD, J. B. 1970. «Pouvoir central et libertés locales. Le monnayage en bronze de Claude avant 50 aprés J.-C.», $R N 12$, 33-61.

GISBERT, J. A. 1987. «La producció de vi al territori de Dianium durant l'Alt Imperi: el taller d'àmfores de la vil.la romana de l'Almadrava (Setla-Mirarosa-Miraflor)», en $E l v i$ a l'Antiquitat. Economia, producciió i comerc al Mediterrani occidental. Actes del I Col.loqui d'Arqueologia romana. Badalona, novembre i desembre 1985, 104-118.

GISBERT, J. A. 1988. «La Almadrava (Setla-Mirarosa-Miraflor), la Marina Alta», Memòries arqueològiques a la Comunitat Valenciana 1984-1985. Valencia, Conselleria de Cultura, Educació i Ciencia, pp. 21-24.

GONZÁLEZ, A y ABASCAL, J. M. «La ocultación monetal de La D'Eula (Crevillente, Alicante)», Lucentum 6, 1987. 183-196.

GURT, J. M ${ }^{\text {a }}$. 1985. Clunia III. Hallazgos monetarios. La romanización de la Meseta norte a través de la circulación monetaria en Clunia. Exc. Arq. en España 145. Madrid.

HILL, P. V. y KENT, J. P. C. 1978. «The bronze coinage of the house of Constantine A.D. 324-346», en Late roman bronze coinage A.D. 324-498 (part II). Londres (rptd.). 
KENT, J. P. C. 1967. «Fel temp reparatio», NC, 83-90.

- 1978. Roman coins. London.

- 1981. The roman imperial coinage VIII: The family of Constantine I, AD 337-364. London.

LAFRANCHI, L. 1949. «La monetazione imperatoria e senatoria di Claudio I durante il quadriennio 41-44 dopo $\mathrm{Cr}^{\circ} »$, RIN 51, 49 ss.

LAUBENHEIMER, F. 1985. La production des amphores en Gaule Narbonnaise. CRHA 66. Paris.

LAUBENHEIMER, F. 1990. Le temps des amphores en Gaule. Paris, Ed. Errance.

LAUBENHEIMER, F. 1990a. Salèlles d'Aude. Un complexe de potiers gallo-romain: le quartier artissanal. Documents d'archéologie française 26. Paris.

LAUBENHEIMER, F. y COURRENT, M. Les potiers de Salelles d'Aude. Paris.

LLOBREGAT, E.A. 1968. «Una aproximación a la circulación monetaria en la costa alicantina antes del cambio de Era", Comunicaciones a la I reunión de historia de la economía antigua de la Península Ibérica. Papeles del Labor. de Arqueol. Valencia (= Saguntum) 5, 91-106.

LRBC (Vid. HILL - KENT y CARSON - KENT).

MATEU Y LLOPIS, F. 1952. «El hallazgo de bronces de Claudio I de la Pobla de Mafumet (Tarragona)», Boletín Arqueológico [Tarragona] 52, 49-53.

MATTINGLY, H. y SYDENHAM, E. A. 1972. The roman imperial coinage II: Vespasian to Hadrian. London (rptd. de la edición de 1926).

- 1930. Id. III: Antoninus Pius to Commodus. London.

- 1972. Id. IV.1: Pertinax to Geta. London (rptd. de la edición de 1936).

MATTINGLY, H. et alii. 1972. The roman imperial coinage IV.2: Macrinus to Pupienus. London (rptd. de la edición de 1938).

- 1949. The roman imperial coinage IV.3: Gordian III - Uranius Antoninus. London.

MEDRANO, M. 1990. Análisis estadístico de la circulación monetaria bajoimperial romana. Zaragoza.
PEREIRA, I. et alii. 1974. Fouilles de Conimbriga III. Les monnaies. Paris.

REECE, R. 1982. «The roman coins», en Wacher, J. y Mc Whirr, A. (ed.), Early roman occupation at Cirencester. Cirencester excavations I. Cirencester.

- 1986. Identifying roman coins. London.

RIC I (Vid. C.H.V. SUTHERLAND 1984).

RIC II, III y IV.1 (Vid. H.B. MATTINGLY y E.A. SYDENHAM).

RIC IV.2 y IV.3 (Vid. H.B. MATTINGLY et alii).

RIC V.1 y V.2 (Vid. P.H. WEBB 1927 y 1933).

RIC VI (Vid. C.H.V. SUTHERLAND 1967).

RIC VII (Vid. P.M. BRUUN 1984).

RIC VIII (Vid. J.P.C. KENT 1981).

RIPOLL, E. et alii. 1979. «Circulación monetaria en Emporion», I Symposium numismático de Barcelona, vol. I, 45 ss.

RIPOLLÉS, P. P. 1980. La circulación monetaria en las tierras valencianas durante la Antigüedad. Barcelona.

- 1982. La circulación monetaria en la Tarraconense mediterránea. Trabajos Varios del SIP 77. Valencia.

SUTHERLAND, C. H. V. 1935. Romano-British imitations of bronze coins of Claudius I. New York.

- 1967. The roman imperial coinage VI: Fron Diocletian's reform ( $A D$ 294) to the death of Maximinus ( $A D$ 313). London.

- 1974. Monnaies romaines. Fribourg.

- 1984. The roman imperial coinage I (revised edition): $3 I$ BC - $A D$ 69. London.

TOYNBEE, J. M. C. 1944. Roman medallions. New York.

VILLARONGA, L. 1979. Numismática antigua de Hispania. Barcelona.

VITTORIO, A. 1985. «Le monete», en A. Carandini et alii, Settefinestre. Una villa schiavistica nell'Etruria romana; vol. 3: La villa e i suoi reperti. Roma, pp. 255-264.

VIVES, A. 1926. La moneda hispánica. Madrid 1980 (rptd.)

WEBB, P. H. 1927. The roman imperial coinage V.I: Valerian to Florian. London.

- 1933. Id. V.2: Probus to Amandus. Loṇdon. 